\title{
Universidade e o ecossistema de inovação: revisão estruturada de literatura
}

\section{University and the innovation ecosystem: structured literature review}

\author{
Gustavo Canaver Pedrinho Mestrando, Universidade Federal de Santa Catarina (UFSC), Brasil - \\ gustavo.pedrinho@outlook.com \\ Danielly Nunes de Carvalho Mestre, Universidade Federal de Santa Catarina (UFSC), Brasil - \\ daniellynunescarvalho@gmail.com \\ Clarissa Stefani Teixeira Doutora, Universidade Federal de Santa Catarina (UFSC), Brasil - clastefani@gmail.com \\ Álvaro Guillermo Rojas Lezana Doutor, Universidade Federal de Santa Catarina (UFSC), Brasil - alvaro.lezana@ufsc.br
}

\section{RESUMO}

As universidades estão tornando-se atores cada vez mais ativos nos ambientes de inovação, agregando valor aos processos de desenvolvimento regional e apoiando a importância do conhecimento no ecossistema de inovação, assim, gerando inovação a partir do conhecimento. Este estudo tem como objetivo investigar a literatura científica sobre o tema universidade e o ecossistema de inovação nos últimos anos. A pesquisa é classificada como descritiva e exploratória. $O$ processo de seleção da coleção de artigos para análise resultou em 34 artigos em consonância com o tema de pesquisa nas bases de dados Web of Science e Scopus. A análise bibliométrica identificou que os artigos foram escritos por 92 autores e coautores, publicados em 30 periódicos no período de 2006 a 2018 em 22 países, 62 instituições de ensino e, apresentam um conjunto de 69 palavraschave. Conclui-se através da análise de conteúdo que as universidades, assumiram um papel mais empreendedor como atores centrais dentro dos ecossistemas de inovação, resultando em novas e diversas oportunidades. Os estudos selecionados mostraram que a colaboração universidade-indústria é a dimensão mais importante de um ecossistema de inovação. No entanto, a literatura também aponta que deve haver maior interação entre governo, indústria e universidades dentro de um contexto inovador. Portanto, isto aponta a necessidade de estudos como este que exploram o papel das universidades no ecossistema de inovação.

Palavras-chave: Universidade. Ecossistema de Inovação. Revisão de Literatura.

\begin{abstract}
Universities are becoming increasingly active players in innovation environments, adding value to regional development processes and supporting the importance of knowledge in the innovation ecosystem, thus generating innovation from knowledge. This study aims to investigate the scientific literature on the university theme and the innovation ecosystem in recent years. The research is classified as descriptive and exploratory. The process of selecting the collection of articles for analysis resulted in 34 articles in line with the research theme in the Web of Science and Scopus databases. The bibliometric analysis identified that the articles were written by 92 authors and coauthors, published in 30 periodicals between 2006 and 2018 in 22 countries, 62 educational institutions, and present a set of 69 keywords. It is concluded through content analysis that universities have taken on a more entrepreneurial role as central players within the innovation ecosystems, resulting in new and diverse opportunities. The selected studies also showed that university-industry collaboration is the most important dimension of an innovation ecosystem. However, the literature also points out that there must be greater interaction between government, industry and universities within an innovative context. Therefore, this points to the need for studies like this that explore the role of universities in the innovation ecosystem.
\end{abstract}

Keywords: University. Innovation Ecosystem. Literature Review. 


\section{INTRODUÇÃO}

O desenvolvimento econômico baseado no conhecimento pode ser realizado através da colaboração entre governo, indústria e universidade (ETZKOWITZ; KLOFSTEN, 2005). As universidades estão se tornando atores cada vez mais ativos neste cenário, agregando valor aos processos de desenvolvimento regional e ancorando a importância do conhecimento no ecossistema de inovação (MARKKULA; KUNE, 2015). Tornandose potencialmente capazes de contribuir para a solução de problemas técnicos e em avanços tecnológicos na indústria, proporcionando o fomento à inovação no meio que se encontram (LOCKETT et al., 2014).

De acordo com Zhang et al. (2013), ideias inovadoras nascem frequentemente de ecossistema de inovação com características de sistema universitário forte, financiamento forte de pesquisa e desenvolvimento $(P \& D)$ e proteção dos direitos de propriedade intelectual. Entretanto, a literatura sobre ecossistemas de inovação, em uma perspectiva econômica regional, pouco tem explorado a relação de troca de conhecimento, principalmente em universidades de pesquisa (HAYTER, 2016).

As pesquisas sobre inovação e empreendedorismo reconhecem o papel importante da universidade e, de alguma forma, a considera como o ator-chave ou de ligação entre todos os atores envolvidos nos ecossistemas (GUERRERO et al., 2016). De acordo com Tomaél e Marteleto (2006), é importante para efetivar a inovação e a colaboração entre empresas e outros tipos de organizações, não se aplicando somente à colaboração dentro, mas também e entre instituições científicas e tecnológicas. Como destacado por Pellegrin (2007), torna-se mais difícil criar o conhecimento necessário para inovar dentro de fronteiras de uma única organização. Neste contexto, é perceptível a variedade de elementos que cercam as relações entre as universidades e o ecossistema de inovação. Logo, este trabalho de pesquisa tem objetivo de investigar na literatura científica o tema "universidade e o ecossistema de inovação" nos últimos anos, por meio de uma revisão de literatura composta por duas etapas: análise bibliométrica e análise de conteúdo.

Além desta introdução o artigo conta com mais quatro seções, sendo a segunda seção o referencial teórico para o embasamento conceitual referente aos temas abordados. A terceira seção a apresentação do procedimento metodológicos utilizados nesta pesquisa. Seguindo com a quarta seção de resultados, tópico este que é dividido em análise bibliométrica e análise de conteúdo. Por fim, a conclusão e as referências bibliográficas deste estudo.

\section{REFERÊNCIAL TEÓRICO}

Esta seção está dividida em dois temas: Ecossistema de inovação e Universidade e o Ecossistema de inovação para o embasamento teórico, por parte do leitor sobre o assunto a ser tratado nesta pesquisa. A inovação se expandiu além dos laboratórios científicos e tecnológicos nas universidades e nas empresas em direção à sociedade com o propósito de gerar inovação e resolver problemas reais (BRANCO; LIMA, 2018). Segundo Etzkowitz e Leydesdorff (2000), além da pesquisa e do ensino, o papel da universidade é apoiar o desenvolvimento econômico, o que fortalece o argumento de que as universidades devem interagir com o ecossistema de inovação. Logo, é visto como importante o estudo do tema "universidade e o ecossistema de inovação".

\subsection{Ecossistema de Inovação}

Inovação é a palavra da sociedade do conhecimento (AUDY, 2017). Definida pela implementação de algo novo ou significativamente melhorado, pode ser caracterizada por inovação de produto, de processo, organizacional ou ainda inovação de marketing (OECD, 2005; BRENNER et al., 2011). Schumpeter (1939), salienta que o conceito de inovação inclui as flutuações de desenvolvimento técnico, exploração de novos mercados e mudanças no método de fornecimento do produto na economia e a motivação do lucro. 
Maes, Debackere e Van Dun, (2011) destacam, do ponto de vista das universidades intensivas em pesquisa, a inovação como produção bem-sucedida e exploração econômica de novos conhecimentos e tecnologias, ou como novas combinações de conhecimento e tecnologia existentes no mercado.

Para Jacobs (1969), a diversidade das pessoas, empresas e culturas nas cidades constituem um terreno fértil para novas ideias e inovações. A construção de sistema de inovação bem-sucedida necessita da dependência mútua e interações dos principais agentes da atividade econômica, podendo operar de forma eficiente a nível regional (GEROLAMO et al., 2008).

De acordo com Akoijam e Krishna (2017), os ecossistemas de inovação evoluem a partir de modelos nacionais de sistemas de inovação, sendo que o ecossistema de inovação é uma concepção do entendimento de ambiente ou ecologia de várias instituições, atores e outros fatores que envolvem a prática de pesquisa e inovação e, não existe um único ator que possa atuar de forma independente (AKOIJAM; KRISHNA, 2017). Logo, o ecossistema de inovação pode ser compreendido também, como um conjunto dinâmico e colaborativo de atores, relações e instituições, que afetam o processo de inovação dentro de uma região (ASHEIM; GERTLER, 2005; ROMANO; PASSIANTE; DEL VECCHIO, 2014).

Os ecossistemas promovem a inter-relação e integração de diferentes conhecimentos fontes e fornecedores (ZAJAC; OSLEN, 1993; POWELL; KOPUT; SMITH, 1996). Nesse processo, universidades e centros de pesquisa, grandes e pequenas empresas, instituições e governos são chamados a colaborar e cooperar entre si, compartilhando conhecimento e trocando experiências, com um envolvimento mais direto e ativo dos usuários em todas as etapas do processo de inovação (DEL VECCHIO, 2017).

\subsection{Universidade e o Ecossistema de Inovação}

Phan e Siegel, (2005) destacam o papel da universidade no ecossistema de inovação como intermediária entre os outros atores, ajudando-os a aprimorar novos conhecimentos e tecnologias. À medida que as tecnologias e novas indústrias estão se tornando mais sofisticadas, as universidades estão desempenhando um papel cada vez mais importante nos processos de invenção, inovação e comercialização (COSH; HUGHES, 2010). O papel das universidades com organizações é chave em diferentes sistemas e, também, tem sido enfatizado por outros autores em suas pesquisas sobre inovação (COOKE; MORGAN, 1998; MALERBA, 2004; EDQUIST, 2005; MOWERY; SAMPAT, 2005).

Nos últimos anos, as universidades assumiram um papel mais empreendedor como atores centrais dentro dos ecossistemas regionais de inovação (ETZKOWITZ; LEYDESDORFF, 2000; ARNKIL et al., 2010). O papel emergente de uma universidade empreendedora moderna é dicotômico, focado tanto na inovação e empreendedorismo que contribui com a competitividade e o desenvolvimento econômico regional (URBANO; GUERRERO, 2013; AUDRESTSCH, 2014).

Durante muito tempo, as duas principais missões das universidades foram o ensino e a pesquisa, havendo uma mudança com a comercialização de pesquisas contribuindo com a inovação, desempenhando assim, um papel mais empreendedor (COSH; HUGHES, 2010; STRIUKOVA; RAYNA, 2015). Audy (2017) destaca, que o papel das universidades como vetor de troca de conhecimento tem avançado cada vez mais nos últimos anos, a ponto de ser considerada por si só, como uma terceira missão das universidades.

A terceira missão tem sido cada vez mais institucionalizada dentro das universidades (LOCKETT; WRIGHT; WILD, 2014). Descrita pelo autor Vorley (2010), na consistência de geração, utilização, aplicação e exploração de conhecimento e outros universitários capacidades fora do ambiente acadêmico, por meio de ações como planejamento estratégico top-down, mudanças na liderança e apoio nas estruturas organizacionais (FUMASOLI; PINHEIRO; STENSKAER, 2014).

$\mathrm{Na}$ criação de valor a economia e a sociedade em geral, espera-se que as universidades se envolvam ativamente com as partes interessadas dos setores privado, público e terceiro setor (GRADY; PRATT, 2000). Guerrero et al., (2016), afirma que o papel da universidade tem sido entendido como um provedor de conhecimento (tecnologia) em seu contexto inovador e uma importante fonte de crescimento econômico. Outros autores argumentam que acelerando a taxa de criação e distribuição de conhecimento e envolvimento 
das universidades com as partes interessadas externas pode-se trazer maior prosperidade econômica (VORLEY; LAWTON, 2007; HOWLETT, 2010).

A interação entre a indústria e a universidade é um forte elo do ecossistema de inovação e gera benefícios para ambos os lados (MAES; DEBACKERE; VAN DUN, 2011). Na visão das empresas, fornecem acesso à tecnologia, conhecimento, talento (CYERT; GOODMAN, 1997), rede de contatos, mão de obra qualificada (recrutamento), pesquisa aplicada e acesso a recursos de conhecimento (LEE, 2010). Quanto às universidades, além do financiamento externo (HARMAN, 2001), as colaborações universidade-indústria podem fornecer dados de pesquisa, aplicação de resultados de pesquisa básica a problemas da indústria (LEE, 2010). Sendo as empresas inovadoras com estruturas relevantes, as mais propensas a colaborar com instituições de ensino superior (SANTORO; CHAKRABARTI, 2002; KRUSS, 2011). Veugelersa e Cassiman (2005), caracterizam o cenário de cooperação em $P \& D$ entre universidade e indústria por altas incertezas devido às assimetrias de informação entre parceiros.

Para Schartinger et al. (2002), as interações entre universidades e indústria podem ser classificadas de acordo com quatro categorias diferentes: pesquisa conjunta (incluindo publicação conjunta), pesquisa contratual (incluindo consultoria, financiamento de assistentes de pesquisa universitária por firmas), mobilidade (movimentação de pessoal entre universidades e firmas), supervisão conjunta e formação (cooperação em educação, formação de pessoal firme em universidades, palestras por pessoal da indústria). A este respeito, D'Este e Patel (2007), consideram que muitos acadêmicos, especialmente aqueles envolvidos em pesquisa aplicada, usam vários tipos simultaneamente.

\section{PROCEDIMENTOS METODOLÓGICOS}

A presente pesquisa é de natureza teórica descritiva e exploratória em relação ao tema abordado, pois trata-se de uma revisão sistemática da literatura (GIL, 2008). A revisão sistemática é uma síntese rigorosa de todas as pesquisas relacionadas com um tópico específico, ela tem como princípios gerais uma busca exaustiva dos estudos analisados, a seleção justificada dos estudos por critérios de inclusão e exclusão explícitos e a avaliação da qualidade metodológica (LIMA; SOARES; BACALTCHUK, 2000; GALVÃO; SAWADA; TREVIZAN, 2004). Tendo como objetivo investigar a literatura científica sobre o tema universidade e o ecossistema de inovação nos últimos anos com base em artigos científicos já publicados.

\subsection{Método de Pesquisa}

O método da pesquisa é composto por duas etapas: análise bibliométrica e análise de conteúdo.

\subsubsection{Etapa 1 - Análise bibliométrica}

A análise bibliométrica foi realizada com o objetivo de identificar e selecionar as principais publicações relevantes ao tema nas bases de dados, juntamente com uma análise quantitativa da produção científica: ano de publicação, autores, países, instituições de ensino, periódicos, palavras-chave, palavras com destaque nos títulos e a redes de colaboração entre os autores.

Como estratégia de busca se iniciou com a definição do tema de pesquisa (Universidade e Ecossistema de Inovação), palavras-chaves (university and innovation ecosystem or ecosystem of innovation) e as bases de dados (Web of Science e Scopus.) No processo de filtragem foram considerados "artigos publicados em periódicos" em todas as áreas. O portfólio bibliográfico foi definido segundo os seguintes critérios: (i) identificação dos artigos repetidos (critério de exclusão); (ii) artigos publicados no idioma inglês e português; (iii) título alinhado ao tema de pesquisa; (iv) resumo alinhado ao tema de pesquisa, (v) disponíveis nas bases de dados (critério de exclusão); e (vi) texto alinhado ao tema de pesquisa (leitura integral). 


\subsubsection{Etapa 2 - Análise conteúdo}

A partir da seleção da literatura que compõem o portfólio bibliográfico foi possível estudar o conteúdo das obras e identificar os principais temas abordados e os assuntos centrais discutidos na área em estudo.

Para o gerenciamento, tabulação e tratamento dos dados coletados, foram utilizados os softwares EndNote ${ }^{\oplus}$ X5, Excel e Vos Viewer. 0 software EndNote ${ }^{\oplus}$ X5 é um gerenciador de referências integrado com as bases de dados facilitando a pesquisa e análise dos artigos científicos (ENDNOTE, 2011). Na análise de coautoria de autores foi utilizado o VosViewer, trata-se de um software que permite construir redes bibliométricas baseadas em dados baixados de bancos de dados bibliográficos (VAZ; LEZANA; MALDONADO, 2017). Para contribuir com a análise de temática dos artigos foi realizado o estudo das palavras com maior destaque nos títulos e palavras-chaves, ou seja, maior número de repetições. A representação visual desta análise foi realizada pelo site "Wordclouds.com".

\section{RESULTADOS}

Nesta seção, apresentam-se os resultados obtidos por meio da aplicação do método de revisão de literatura apresentado no capítulo anterior, que está dividido em dois momentos: i) análise bibliométrica e ii) análise de conteúdo.

\subsection{Análise bibliométrica}

$\mathrm{Na}$ fase de busca e filtragem bibliométrica como o tema de pesquisa "Universidade e Ecossistema de Inovação" foram encontrados 101 artigos nas bases de dados Web of Science e Scopus. A busca foi realizada de modo a combinar as palavras-chave conforme o quadro abaixo e encontrar as publicações que contêm as palavras-chave no Abstract, Title ou Keywords e sem delimitação temporal possibilitando verificar a evolução do tema durante os anos.

Quadro 1 - Definição das palavras-chave

\begin{tabular}{|l|c|}
\hline \multicolumn{2}{|c|}{ Palavras-Chave } \\
\hline universit*... AND & "innovation ecosystem *" \\
\cline { 2 - 2 } & "ecosystem* of innovation" \\
\hline
\end{tabular}

Fonte: Elaborado pelos autores (2019).

O símbolo de truncamento "*" foi usado para ampliar o número de respostas, pois este recupera plurais, expressões com o mesmo radical e variações de grafia da palavra-chave. Com os critérios utilizados nesta pesquisa e exposto na seção de procedimentos metodológicos, apenas 34 artigos se encontram alinhados ao tema em estudo para compor o portfólio bibliográfico (PB) (Quadro 1). A Figura 1 apresenta a quantidade de artigos retornados na busca, as etapas de triagem dos artigos e da análise sistemática que foi adotada. 
Figura 1 - Detalhamento da Etapa 1 e o número de artigos retornados em cada etapa

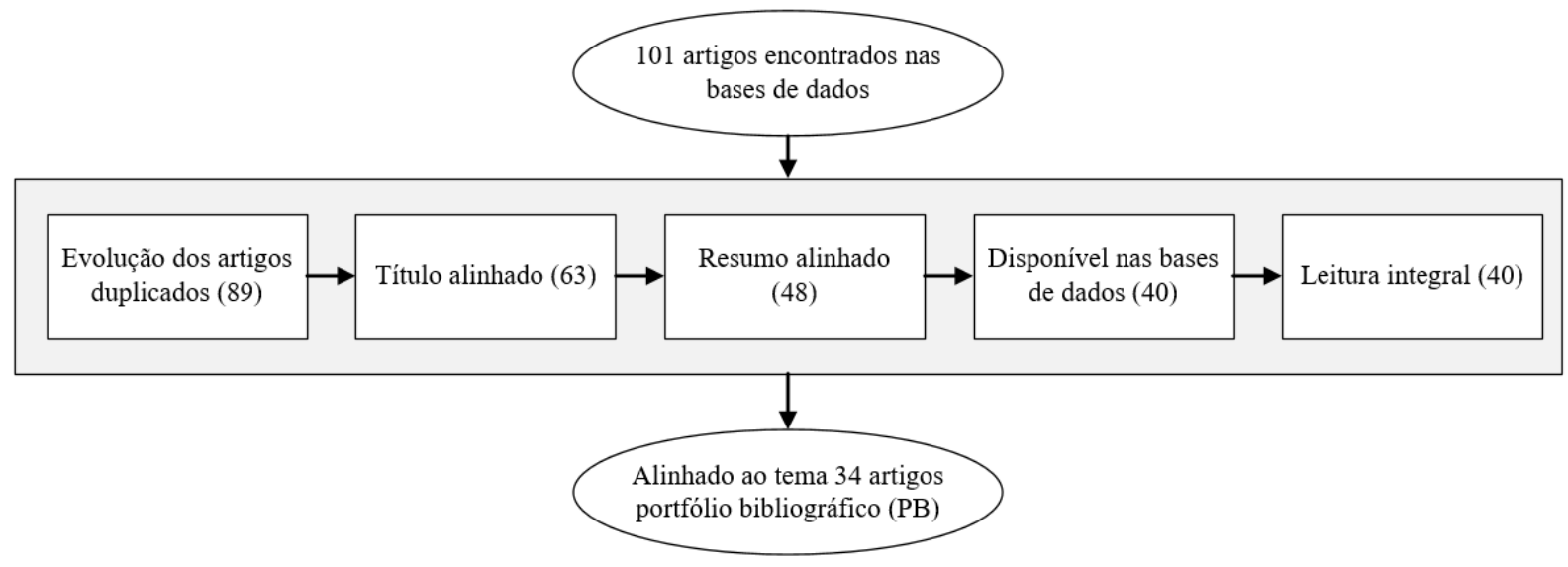

Fonte: Elaborado pelos autores

O portfólio bibliográfico abaixo inclui o ano da publicação, autor/autores e título do artigo e periódico apresentados em ordem cronológica, Quadro 2.

Quadro 2 - Portfólio bibliográfico

\begin{tabular}{|c|c|c|c|}
\hline Ano & Autores & Título & Periódico \\
\hline 2018 & BRANCO, Jose; LIMA, Celso. & $\begin{array}{l}\text { Universities as knowledge flow-based } \\
\text { innovation ecosystems }\end{array}$ & $\begin{array}{l}\text { Revista Praxis } \\
\text { Educacional }\end{array}$ \\
\hline 2018 & $\begin{array}{l}\text { THEODORAKI, Christina; } \\
\text { MESSEGHEM, Karim; RICE, } \\
\text { P. }\end{array}$ & $\begin{array}{c}\text { A social capital approach to the } \\
\text { development of sustainable entrepreneurial } \\
\text { ecosystems: an explorative study }\end{array}$ & $\begin{array}{l}\text { Small Business } \\
\text { Economics }\end{array}$ \\
\hline 2017 & AUDY, Jorge. & $\begin{array}{c}\text { A inovação, o desenvolvimento e o papel da } \\
\text { Universidade }\end{array}$ & Estudos Avançados \\
\hline 2017 & CHEN, Shih; LIN, Wei. & $\begin{array}{c}\text { The dynamic role of universities in } \\
\text { developing an emerging sector: a case } \\
\text { study of the biotechnology sector }\end{array}$ & $\begin{array}{l}\text { Technological } \\
\text { Forecasting and Social } \\
\text { Change }\end{array}$ \\
\hline 2017 & $\begin{array}{l}\text { DANIELS, Chux; } \\
\text { USTYUZHANTSEVA, Olga; } \\
\text { YAO, Wei. }\end{array}$ & $\begin{array}{c}\text { Innovation for inclusive development, } \\
\text { public policy support and triple helix: } \\
\text { perspectives from BRICS }\end{array}$ & $\begin{array}{c}\text { African Journal of Science } \\
\text { Technology Innovation \& } \\
\text { Development }\end{array}$ \\
\hline 2017 & $\begin{array}{l}\text { DEL VECCHIO, Pasquale et } \\
\text { al. }\end{array}$ & $\begin{array}{l}\text { Living Lab as an Approach to Activate } \\
\text { Dynamic Innovation Ecosystems and } \\
\text { Networks: An Empirical Study }\end{array}$ & $\begin{array}{l}\text { International Journal of } \\
\text { Innovation and } \\
\text { Technology Management }\end{array}$ \\
\hline 2017 & $\begin{array}{c}\text { GALAN-MUROS, Victoria et } \\
\text { al. }\end{array}$ & $\begin{array}{c}\text { Nurture over nature: How do European } \\
\text { universities support their collaboration with } \\
\text { business? }\end{array}$ & $\begin{array}{l}\text { Journal of Technology } \\
\text { Transfer }\end{array}$ \\
\hline 2017 & $\begin{array}{l}\text { HU, Lina; LI, Yali; ZHANG, } \\
\text { Bin. }\end{array}$ & $\begin{array}{c}\text { Study on university scientific innovation and } \\
\text { cultural construction from the perspective } \\
\text { of public management }\end{array}$ & $\begin{array}{l}\text { Revista de la Facultad de } \\
\text { Ingenieria }\end{array}$ \\
\hline 2017 & $\begin{array}{c}\text { KIVIMAA, Paula; BOON, } \\
\text { Wouter; ANTIKAINEN, Riina. }\end{array}$ & $\begin{array}{c}\text { Commercialising university inventions for } \\
\text { sustainability-a case study of (non- } \\
\text { )intermediating 'cleantech' at Aalto } \\
\text { University }\end{array}$ & $\begin{array}{l}\text { Science and Public } \\
\text { Policy }\end{array}$ \\
\hline 2017 & $\begin{array}{l}\text { RANGA. Marina; } \\
\text { MROCZOWSKI, Tomasz; } \\
\text { ARAISO, Tsunehisa. }\end{array}$ & $\begin{array}{c}\text { University-industry cooperation and the } \\
\text { transition to innovation ecosystems in } \\
\text { Japan }\end{array}$ & $\begin{array}{l}\text { Industry and Higher } \\
\text { Education }\end{array}$ \\
\hline
\end{tabular}




\begin{tabular}{|c|c|c|c|}
\hline 2017 & $\begin{array}{l}\text { ZHOU, Liqing; ZHANG, } \\
\text { Suodi; AN, Young. }\end{array}$ & $\begin{array}{c}\text { Actor-network theory application in global } \\
\text { power city: An empirical study }\end{array}$ & $\begin{array}{l}\text { Revista de la Facultad de } \\
\text { Ingenieria }\end{array}$ \\
\hline 2016 & BROWN, Roger. & $\begin{array}{c}\text { Mission impossible? Entrepreneurial } \\
\text { universities and peripheral regional } \\
\text { innovation systems }\end{array}$ & Industry and Innovation \\
\hline 2016 & $\begin{array}{l}\text { EGLASH, Stephen; RIZK, } \\
\text { Sarah. }\end{array}$ & $\begin{array}{l}\text { Discovery and application of exemplary } \\
\text { models of innovation }\end{array}$ & MRS Bulletin \\
\hline 2016 & GUERRERO, Maribel et al. & $\begin{array}{c}\text { Entrepreneurial universities: emerging } \\
\text { models in the new social and economic } \\
\text { landscape }\end{array}$ & $\begin{array}{l}\text { Small Business } \\
\text { Economics }\end{array}$ \\
\hline 2016 & HAYTER, Chistopher. & $\begin{array}{l}\text { A trajectory of early-stage spinoff success: } \\
\text { the role of knowledge intermediaries within } \\
\text { an entrepreneurial university ecosystem }\end{array}$ & $\begin{array}{l}\text { Small Business } \\
\text { Economics }\end{array}$ \\
\hline 2016 & $\begin{array}{l}\text { MCADAM, Maura; MILLER, } \\
\text { Kristel; MCADAM, Rodney. }\end{array}$ & $\begin{array}{l}\text { Situated regional university incubation: } A \\
\text { multi-level stakeholder perspective }\end{array}$ & Technovation \\
\hline 2016 & MILLER, Kristel et al. & $\begin{array}{c}\text { Knowledge transfer in university quadruple } \\
\text { helix ecosystems: An absorptive capacity } \\
\text { perspective }\end{array}$ & R\&D Management \\
\hline 2016 & RANGA, Marina et al. & $\begin{array}{l}\text { Building Technology Transfer Capacity in } \\
\text { Turkish Universities: A critical analysis }\end{array}$ & $\begin{array}{l}\text { European Journal of } \\
\text { Education }\end{array}$ \\
\hline 2016 & $\begin{array}{l}\text { ROSLI, Ainurul; ROSSI, } \\
\text { Federica. }\end{array}$ & $\begin{array}{c}\text { Third-mission policy goals and incentives } \\
\text { from performance-based funding: Are they } \\
\text { aligned? }\end{array}$ & Research Evaluation \\
\hline 2016 & UGNICH, Ekaterina et al. & $\begin{array}{c}\text { University innovation ecosystem as a } \\
\text { mechanism of innovation process } \\
\text { development }\end{array}$ & $\begin{array}{l}\text { Social Sciences } \\
\text { (Pakistan) }\end{array}$ \\
\hline 2015 & $\begin{array}{l}\text { MARKKULA, Markku; KUNE, } \\
\text { Hank. }\end{array}$ & $\begin{array}{c}\text { Making Smart Regions Smarter: Smart } \\
\text { Specialization and the Role of Universities in } \\
\text { Regional Innovation Ecosystems }\end{array}$ & $\begin{array}{l}\text { Technology Innovation } \\
\text { Management Review }\end{array}$ \\
\hline 2015 & $\begin{array}{l}\text { STRIUKOVA, Ludmila; } \\
\text { RAYNA, Thierry. }\end{array}$ & $\begin{array}{c}\text { University-industry knowledge exchange: } \\
\text { An exploratory study of Open Innovation in } \\
\text { UK universities }\end{array}$ & $\begin{array}{c}\text { European Journal of } \\
\text { Innovation Management }\end{array}$ \\
\hline 2014 & $\begin{array}{l}\text { CHANDRAN, Govindaraju; } \\
\text { SUNDRAM, Veera; } \\
\text { SANTHIDRAN, Sinnappan. }\end{array}$ & $\begin{array}{c}\text { Innovation systems in Malaysia: } A \\
\text { perspective of university-industry } R \& D \\
\text { collaboration }\end{array}$ & Al and Society \\
\hline 2014 & $\begin{array}{l}\text { MARTINEZ, Saez; MORENO, } \\
\text { Gonzalez; HOGAN, Teresa. }\end{array}$ & $\begin{array}{l}\text { The role of university in eco- } \\
\text { entrepreneurship: Evidence from the } \\
\text { eurobarometer survey on attitudes of } \\
\text { european entrepreneurs towards eco- } \\
\text { innovation }\end{array}$ & $\begin{array}{l}\text { Environmental } \\
\text { Engineering and } \\
\text { Management Journal }\end{array}$ \\
\hline 2013 & BRODHAG, Christian. & $\begin{array}{c}\text { Research universities, technology transfer, } \\
\text { and job creation: what infrastructure, for } \\
\text { what training? }\end{array}$ & $\begin{array}{l}\text { Studies in Higher } \\
\text { Education }\end{array}$ \\
\hline 2013 & ETZKOWITZ, Henry. & $\begin{array}{l}\text { StartX and the 'Paradox of Success': Filling } \\
\text { the gap in Stanford's entrepreneurial culture }\end{array}$ & $\begin{array}{l}\text { Social Science } \\
\text { Information }\end{array}$ \\
\hline 2013 & ZHANG, Linxiu et al. & $\begin{array}{l}\text { Are elite university graduates aiding China's } \\
\text { transition to an innovation-based economy? } \\
\text { Results from a career choices survey among } \\
\text { would-be innovators in China and the USA }\end{array}$ & $\begin{array}{l}\text { Asia-Pacific Journal of } \\
\text { Accounting and } \\
\text { Economics }\end{array}$ \\
\hline
\end{tabular}




\begin{tabular}{|c|c|c|c|}
\hline 2012 & HARRISON, Kenneth et al. & $\begin{array}{l}\text { Building a life sciences innovation } \\
\text { ecosystem }\end{array}$ & $\begin{array}{l}\text { Science Translational } \\
\text { Medicine }\end{array}$ \\
\hline 2011 & $\begin{array}{l}\text { CARAYANNIS, Elias; } \\
\text { CAMPBELL, David. }\end{array}$ & $\begin{array}{c}\text { Open Innovation Diplomacy and a 21st } \\
\text { Century Fractal Research, Education and } \\
\text { Innovation (FREIE) Ecosystem: Building on } \\
\text { the Quadruple and Quintuple Helix } \\
\text { Innovation Concepts and the "Mode 3" } \\
\text { Knowledge Production System }\end{array}$ & $\begin{array}{l}\text { Journal of the Knowledge } \\
\text { Economy }\end{array}$ \\
\hline 2011 & $\begin{array}{l}\text { MAES, Katrien; DEBACKERE, } \\
\text { Koenraad; VAN DUN, Paul. }\end{array}$ & $\begin{array}{l}\text { Universities, research and the "innovation } \\
\text { Union" }\end{array}$ & $\begin{array}{l}\text { Procedia - Social and } \\
\text { Behavioral Sciences }\end{array}$ \\
\hline 2011 & $\begin{array}{l}\text { MERCAN, Birol; GOKTAS, } \\
\text { Din. }\end{array}$ & $\begin{array}{l}\text { Components of innovation ecosystems: } A \\
\text { cross-country study }\end{array}$ & $\begin{array}{c}\text { International Research } \\
\text { Journal of Finance and } \\
\text { Economics }\end{array}$ \\
\hline 2011 & $\begin{array}{l}\text { TRAILER, Helmut; WATZKE, } \\
\text { Heribert; SAGUY, Sam. }\end{array}$ & $\begin{array}{c}\text { Reinventing R\&D in an Open Innovation } \\
\text { Ecosystem }\end{array}$ & Journal of Food Science \\
\hline 2010 & $\mathrm{COSH}$, Andy; HUGHES, Alan. & $\begin{array}{c}\text { Never mind the quality feel the width: } \\
\text { University-industry links and government } \\
\text { financial support for innovation in small } \\
\text { high-technology businesses in the UK and } \\
\text { the USA }\end{array}$ & $\begin{array}{l}\text { Journal of Technology } \\
\text { Transfer }\end{array}$ \\
\hline 2006 & SMITH, Kenneth. & $\begin{array}{c}\text { Building an Innovation Ecosystem: Process, } \\
\text { Culture and Competencies }\end{array}$ & $\begin{array}{l}\text { Industry and Higher } \\
\text { Education }\end{array}$ \\
\hline
\end{tabular}

Fonte: Elaborado pelos autores (2019).

Os artigos foram analisados procurando identificar: número de artigo por autor, publicações ao longo dos anos, países, instituições de ensino, número de publicações por periódicos, as palavras-chave mais presentes nas publicações, os termos com maior destaque nos títulos dos artigos e a rede de colaboração entre os autores.

Os 34 artigos foram escritos por 92 autores e coautores, publicado em 30 periódicos, no período de 2006 a 2018, em 22 países com destaque aos USA com 11 publicações na área (Figura 2), 62 instituições de ensino e 69 palavras-chave.

Figura 2 - Países com publicações no tema

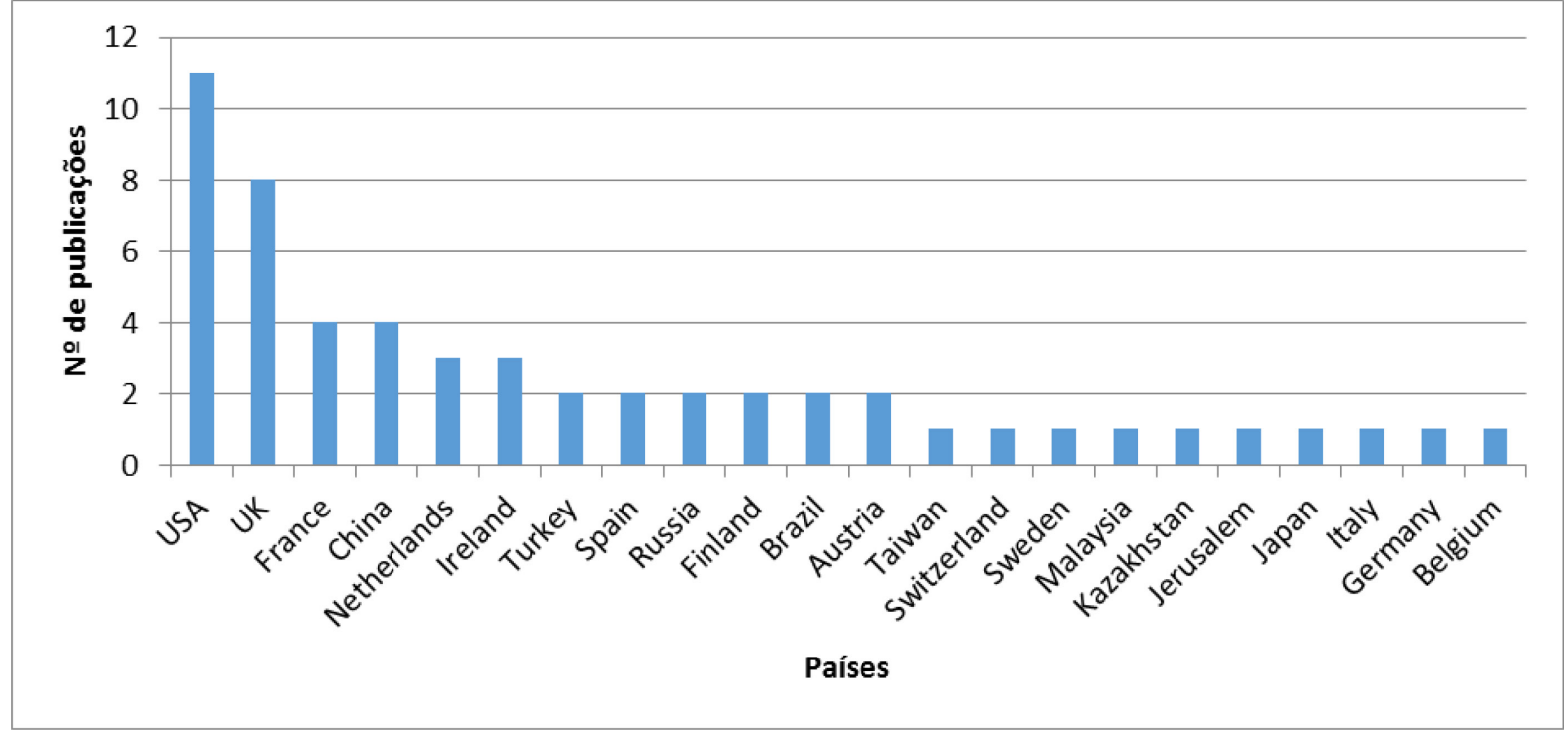

Fonte: Elaborado pelos autores (2019). 
Observa-se na Figura 3 a distribuição cronológica dos artigos publicados no tema e o acumulado por ano. Verifica-se que a primeira publicação ocorreu em 2006, havendo um período de quatro anos até a segunda publicação e, ainda que a maior concentração esteja nos anos 2016 e 2017 com nove publicações cada ano.

Figura 3 - Ordem cronológica de publicações

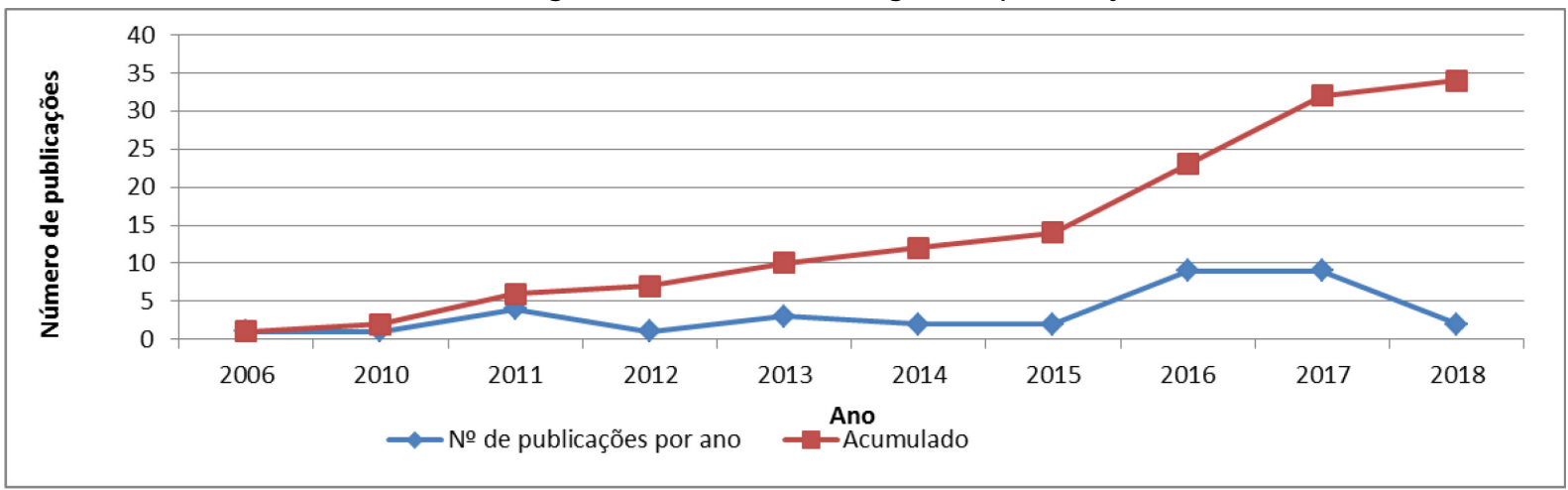

Fonte: Elaborado pelos autores (2019).

Analisando os autores mais relevantes do PB com auxílio do software VosViewer, identificou-se 92 autores e coautores que juntos formam 32 clusters com 114 links. Os clusters representam os autores que trabalharam juntos em seus estudos de pesquisa e os links são as conexões, ou seja, relação de coautoria, conforme Figura 4.

Figura 4 - Rede de cooperação entre autores smith, k. r.

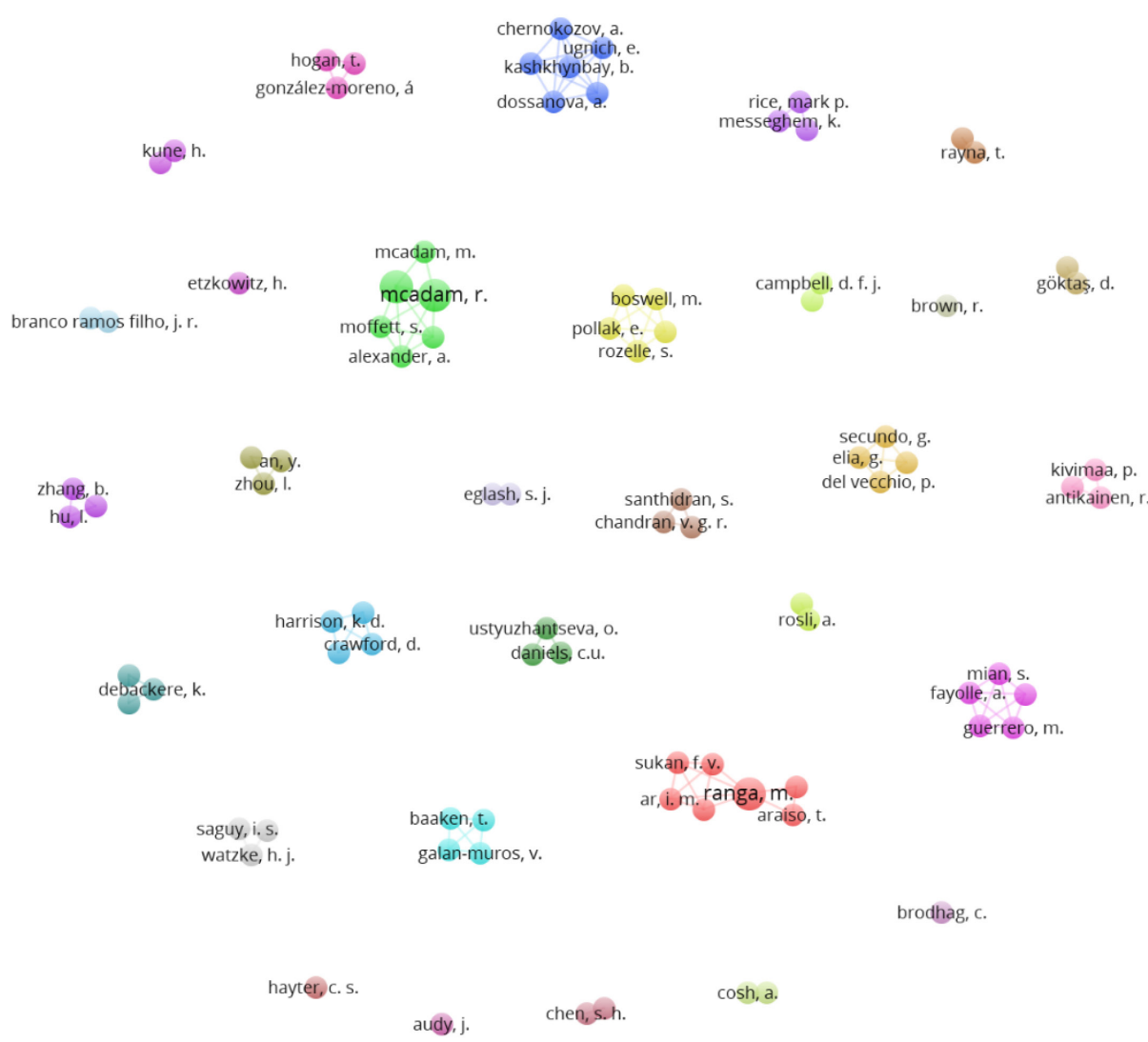

Fonte: Elaborado pelos autores (2019). 
Com a rede de autores é possível identificar uma segmentação na área, simbolizados pelo alto número de pequenos clusters (menos de quatro autores). Em geral, os autores e coautores estão abordando o mesmo tema central 'universidade e o ecossistema de inovação", porém os assuntos abordados seguem com diferentes direcionamentos, por exemplo: universidade-indústria, quádrupla hélice, universidade empreendedora, entre outros. Logo, isso evidencia o alto número de clustere poucos links. Os maiores clusters estão em vermelho, verde, azul, amarelo e roxo, sendo compostos por mais de cinco autores, conforme Quadro 3.

Quadro 3 - Detalhamento dos maiores clusters de autores e coautores

\begin{tabular}{|c|c|c|c|}
\hline Cluster & Autor & Instituição de ensino & País \\
\hline \multirow{7}{*}{$\begin{array}{l}\text { Vermelho } \\
\text { (1) }\end{array}$} & Ar,I.M. & Karadeniz Technical University & Turkey \\
\hline & Araiso,T. & Hokkaido University & Japan \\
\hline & Mroczkowski,T. & American University & USA \\
\hline & Ranga, M. & Stanford University & USA \\
\hline & Sukan, F.V. & Ege University & Turkey \\
\hline & Temel, S. & Ege University & Turkey \\
\hline & Yesilay, R.B. & Ege University & Turkey \\
\hline \multirow[t]{6}{*}{ Verde (2) } & Alexander,A. & University of Exeter & UK \\
\hline & McAdam, M. & Ulster University & UK \\
\hline & McAdam, R. & Ulster University & UK \\
\hline & Miller,K. & Queen's University & Ireland \\
\hline & Moffett,S. & Ulster University & UK \\
\hline & Puthusserry,P. & Queen's University & Ireland \\
\hline \multirow{6}{*}{ Azul (3) } & Chernokozov, A. & Don State Technical University & Rússia \\
\hline & Dossanova, A. & Turan Astana University & Rússia \\
\hline & Kashkhynbay, B. & & Rússia \\
\hline & Koryakovtseva,O. & $\begin{array}{l}\text { Yaroslavl State pedagogical } \\
\text { University }\end{array}$ & Rússia \\
\hline & Ugnich, E. & Don State Technical University & Rússia \\
\hline & Velichko, E. & Don State Technical University & Rússia \\
\hline \multirow[t]{5}{*}{ Amarelo (4) } & Boswell, M. & Stanford University & USA \\
\hline & Darwin, $\mathrm{R}$. & Stanford University & USA \\
\hline & Pollak, E. & Stanford University & USA \\
\hline & Rozelle, S. & Stanford University & USA \\
\hline & Zhang, L. & $\begin{array}{l}\text { Institute of Geographical } \\
\text { Sciences and Natural Resources } \\
\text { Research }\end{array}$ & China \\
\hline \multirow[t]{5}{*}{ Roxo (5) } & Fayolle, A. & $\begin{array}{l}\text { Universitat Autonoma de } \\
\text { Barcelona }\end{array}$ & Spain \\
\hline & Guerrero, M. & University of Deusto & Spain \\
\hline & Klofsten, M. & Linkoping University/CIE & Sweden \\
\hline & Mian, S. & $\begin{array}{l}\text { School of Business, State } \\
\text { University of New York }\end{array}$ & USA \\
\hline & Urbano, D. & $\begin{array}{l}\text { Universitat Autonoma de } \\
\text { Barcelona }\end{array}$ & Spain \\
\hline
\end{tabular}

Fonte: Elaborado pelos autores (2019).

Verificou-se nos artigos que apenas três dos autores possuem duas publicações, sendo que dois são coautores do mesmo artigo, os demais autores e coautores possuem somente uma publicação cada no tema. Os autores com duas publicações são Miller,K. e McAdam,R. com os artigos intitulados Knowledge transfer in university quadruple helix ecosystems: An absorptive capacity perspective e Situated regional university 
incubation: A multi-level stakeholder perspective. O terceiro autor com duas publicações é Ranga, M. com os artigos Building Technology Transfer Capacity in Turkish Universities: A critical analysis e University-industry cooperation and the transition to innovation ecosystems in Japan. Os dois maiores clusters (vermelho e verde) são compostos pelos autores com duas publicações e os coautores, conforme pode ser observado com maior detalhe na Figura 5.

Figura 5 - Rede de cooperação dos dois maiores clusters
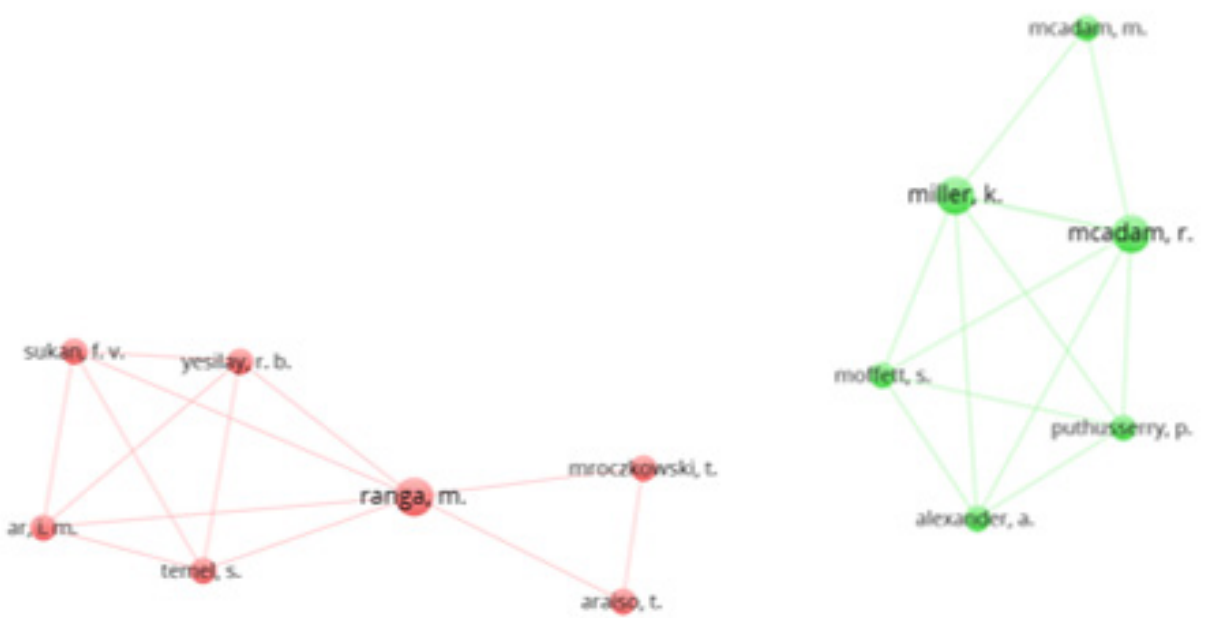

Fonte: Elaborado pelos autores (2019).

Foram identificados um total de 30 periódicos, o periódico Small Business Economics possui três publicações, outros quatro periódicos com duas publicações e os demais apenas um artigo no tema. Na Figura 6, encontra-se os periódicos com mais de duas publicações no tema. O periódico Small Business Economics é um periódico internacional que aborda temas importantes na área de empreendedorismo, empresas familiares, pequenas e médias empresas e criação de novos empreendimentos.

A revista tem um escopo amplo, incluindo características dos empreendedores, áreas ocupacionais, novos empreendimentos e inovação, gestão do ciclo de vida e avaliação de desempenho de empresas, bem como o papel desempenhado pelas instituições e políticas públicas nos contextos locais, regional, nacional e internacional. Os artigos publicados no Small Business Economics tipicamente enfatizam a relevância econômica e social dos resultados da pesquisa, sendo uma revista líder em empreendedorismo, publica artigos teóricos e empíricos, incentivando a pesquisa interdisciplinar de um amplo espectro de disciplinas e áreas.

Figura 6 - Periódicos com publicações no tema

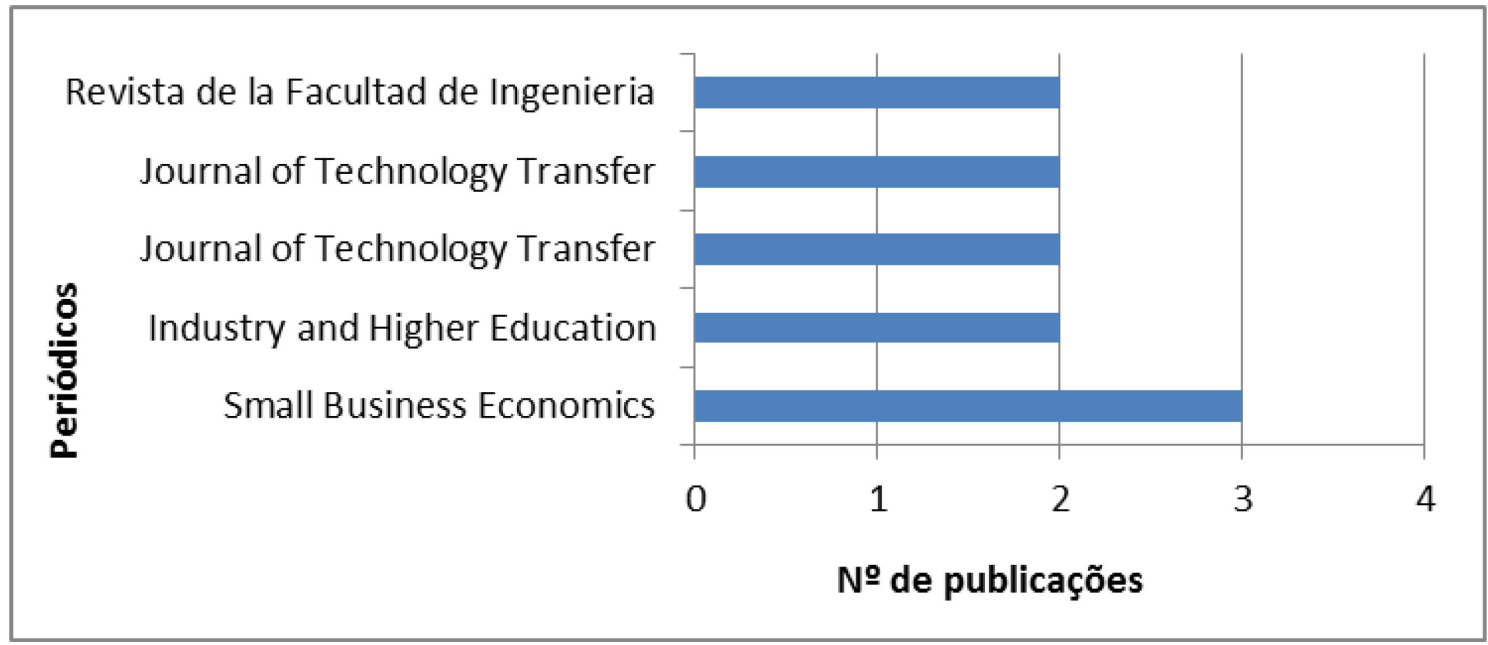

Fonte: Elaborado pelos autores (2019). 
Referente à análise das palavras-chave mais relevantes nos artigos analisados (total de 69 palavraschaves), há uma presença forte das palavras innovation ecosystem (8), innovation (5), innovation policy (2), Triple helix (2), University (2). Uma vez que as palavras em destaque são aquelas que mais se repetem e representam o escopo das temáticas de cada artigo.

Analisou-se também a representatividade das palavras e, não somente dos termos usados como palavras-chave, assim, identificou-se que "University" possui uma representatividade maior, pois como palavra-chave ela é usada em combinação, exemplos: Research Universities, University incubation models, University spinoffs, University-business cooperation, University-industry collaboration e University-industry links. O mesmo acontece com "innovation" que também é associada com outras palavras como: Innovation Entrepreneurship, Innovation networks, open innovation e outros. Na Figura 7 observa-se a representatividade das palavras-chaves.

Figura 7 - Palavras-chaves em destaque

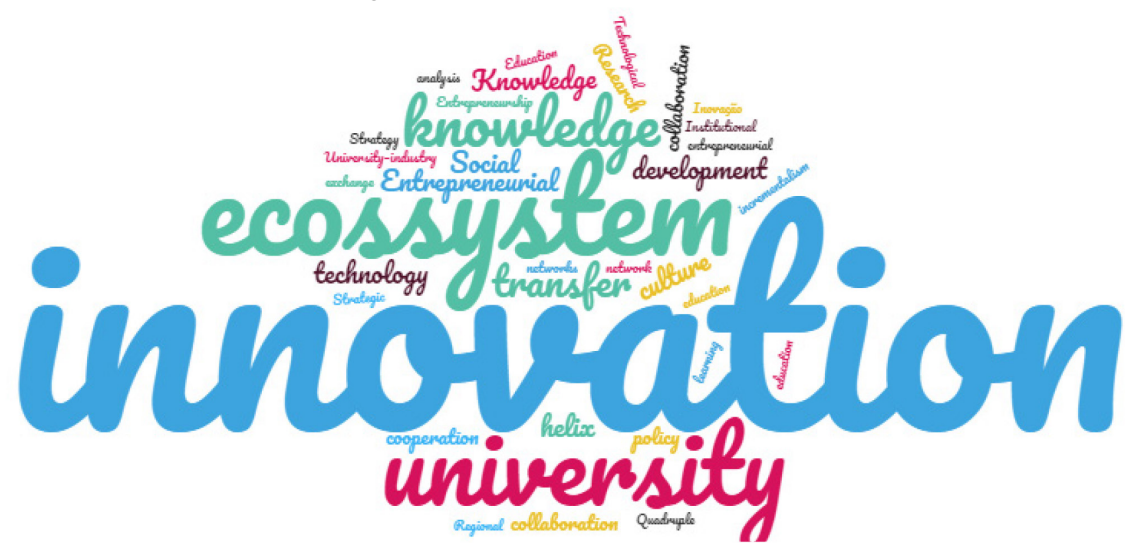

Fonte: Elaborado pelos autores (2019).

Dando continuidade à análise de temática dos artigos, evidenciaram-se também as palavras mais relevantes nos títulos dos dos mesmos, destacando a presença forte dos termos: innovation university $e$ ecosystem. A representação em nuvem de palavras foi realizada por meio do site "Wordclouds.com" permitindo um reconhecimento visual dos termos com maior destaque; a análise foi feita com base no número de vezes que as palavras aparecem no título (Figura 8).

Figura 8 - Palavras em destaque nos títulos

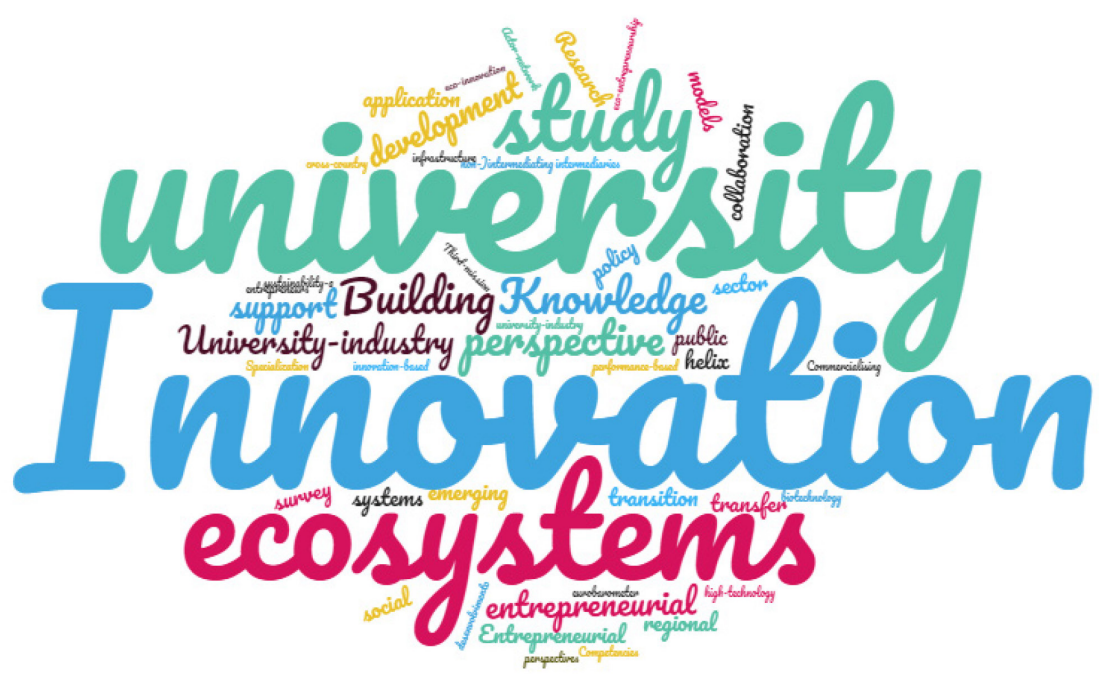

Fonte: Elaborado pelos autores (2019). 
Comparando a Figura 7 e 8 observa-se que Innovation continua com maior representatividade, como também University, nos títulos dos artigos possui mais destaque do que ecosystems quando usado como palavra-chave, portanto são as temáticas mais abordadas nos artigos analisados como esperado devido a busca realizada.

\subsection{Análise de Conteúdo}

$\mathrm{Na}$ etapa de análise de conteúdo a partir da seleção da literatura foi possível levantar os temas abordados pelos artigos do portfólio bibliográfico e uma visão geral dos assuntos centrais discutido na área em estudo.

No Quadro 4, empregou-se análise de conteúdo com a leitura integral das obras identificando os temas abordados de cada artigo do PB.

Quadro 4 - Temas abordados nos artigos do portfólio bibliográfico

\begin{tabular}{|c|c|c|}
\hline Autores & Título & Temas abordados \\
\hline $\begin{array}{l}\text { BRANCO, Jose; LIMA, } \\
\text { Celso. }\end{array}$ & $\begin{array}{l}\text { Universities as knowledge } \\
\text { flow based innovation } \\
\text { ecosystems }\end{array}$ & $\begin{array}{l}\text { Aborda o ecossistema de inovação baseado em fluxos } \\
\text { de conhecimento ao contexto universitário com a } \\
\text { simulação do comportamento de indivíduos em um } \\
\text { ambiente universitário expostos a um ambiente } \\
\text { estável e a um ambiente em constante mudança para } \\
\text { avaliar a evolução de sua motivação em aprender e } \\
\text { adotar novas práticas pedagógicas. }\end{array}$ \\
\hline $\begin{array}{l}\text { THEODORAKI, } \\
\text { Christina; } \\
\text { MESSEGHEM, Karim; } \\
\text { RICE, P. }\end{array}$ & $\begin{array}{c}\text { A social capital approach to } \\
\text { the development of } \\
\text { sustainable entrepreneurial } \\
\text { ecosystems: an explorative } \\
\text { study }\end{array}$ & $\begin{array}{c}\text { Procura desenvolver uma compreensão mais robusta } \\
\text { de ecossistemas empresariais sustentáveis baseados } \\
\text { na universidade, por meio de uma análise } \\
\text { aprofundada de um elemento essencial de apoio ao } \\
\text { empreendedorismo: a incubadora de empresas } \\
\text { universitárias. }\end{array}$ \\
\hline AUD & $\begin{array}{c}\text { A inovação, o } \\
\text { desenvolvimento e o papel } \\
\text { da Universidade }\end{array}$ & $\begin{array}{l}\text { Aborda a temática da relação entre inovação, o } \\
\text { desenvolvimento e o papel da universidade no } \\
\text { contexto da sociedade do conhecimento. }\end{array}$ \\
\hline CHEN, Shih; LIN, Wei. & $\begin{array}{c}\text { The dynamic role of } \\
\text { universities in developing an } \\
\text { emerging sector: a case } \\
\text { study of the biotechnology } \\
\text { sector }\end{array}$ & $\begin{array}{l}\text { Explora as redes de colaboração de P\&D entre as } \\
\text { universidades e os outros atores do sistema de } \\
\text { inovação setorial de biotecnologia para entender } \\
\text { como as universidades fazem uso dos conhecimentos } \\
\text { trocados com outras partes para moldar a sociedade. }\end{array}$ \\
\hline $\begin{array}{l}\text { DANIELS, Chux; } \\
\text { USTYUZHANTSEVA, } \\
\text { Olga; YAO, Wei. }\end{array}$ & $\begin{array}{l}\text { Innovation for inclusive } \\
\text { development, public policy } \\
\text { support and triple helix: } \\
\text { perspectives from BRICS }\end{array}$ & $\begin{array}{l}\text { Investiga o papel da inovação referido como inovação } \\
\text { para o desenvolvimento inclusivo (IID) e os links para } \\
\text { políticas públicas nos países do BRICS (Brasil, Rússia, } \\
\text { Índia, China e África do Sul). }\end{array}$ \\
\hline $\begin{array}{l}\text { DEL VECCHIO, } \\
\text { Pasquale et al. }\end{array}$ & $\begin{array}{c}\text { Living Lab as an Approach to } \\
\text { Activate Dynamic } \\
\text { Innovation Ecosystems and } \\
\text { Networks: An Empirical } \\
\text { Study }\end{array}$ & $\begin{array}{l}\text { Discute a importância do Living Labs como um } \\
\text { mecanismo efetivo que pode apoiar a criação de } \\
\text { ecossistemas e redes dinâmicas de inovação. } \\
\text { Explorando sua relevância para a definição de } \\
\text { estratégias regionais de crescimento inteligente, que } \\
\text { valorizem o conhecimento interno e externo ativos. }\end{array}$ \\
\hline $\begin{array}{l}\text { GALAN-MUROS, } \\
\text { Victoria et al. }\end{array}$ & $\begin{array}{l}\text { Nurture over nature: How do } \\
\quad \text { European universities }\end{array}$ & $\begin{array}{l}\text { Identificar e avaliar os mecanismos que as } \\
\text { universidades europeias estão usando para fomentar a }\end{array}$ \\
\hline
\end{tabular}




\begin{tabular}{|c|c|c|}
\hline & $\begin{array}{c}\text { support their collaboration } \\
\text { with business? }\end{array}$ & $\begin{array}{c}\text { colaboração da indústria em nível estratégico e } \\
\text { operacional e avalia sua relação com as atividades da } \\
\text { UBC (university-business cooperation). }\end{array}$ \\
\hline $\begin{array}{l}\text { HU, Lina; LI, Yali; } \\
\text { ZHANG, Bin. }\end{array}$ & $\begin{array}{c}\text { Study on university scientific } \\
\text { innovation and cultural } \\
\text { construction from the } \\
\text { perspective of public } \\
\text { management }\end{array}$ & $\begin{array}{l}\text { O artigo investiga a integração da cultura de inovação, } \\
\text { construção da cultura inovadora em universidades, na } \\
\text { perspectiva da gestão pública e finaliza com } \\
\text { recomendações políticas para promover a inovação } \\
\text { colaborativa em universidades. }\end{array}$ \\
\hline $\begin{array}{l}\text { KIVIMAA, Paula; } \\
\text { BOON, Wouter; } \\
\text { ANTIKAINEN, Riina. }\end{array}$ & $\begin{array}{l}\text { Commercialising university } \\
\text { inventions for sustainability- } \\
\text { a case study of (non- } \\
\text { Jintermediating 'cleantech' } \\
\text { at Aalto University }\end{array}$ & $\begin{array}{l}\text { Intermediação na fase de pré-comercialização de } \\
\text { invenções de tecnologia limpa desenvolvidas em uma } \\
\text { Universidade da Finlândia, com foco nas atividades de } \\
\text { um intermediário de inovação universitária. }\end{array}$ \\
\hline $\begin{array}{l}\text { RANGA. Marina; } \\
\text { MROCZOWSKI, } \\
\text { Tomasz; ARAISO, } \\
\text { Tsunehisa. }\end{array}$ & $\begin{array}{l}\text { University-industry } \\
\text { cooperation and the } \\
\text { transition to innovation } \\
\text { ecosystems in Japan }\end{array}$ & $\begin{array}{l}\text { Políticas de colaboração universidade-indústria (UIC) } \\
\text { no Japão desde meados da década de } 1990 \text { até o } \\
\text { presente e analisa seu papel na formação do } \\
\text { ecossistema de inovação do país. }\end{array}$ \\
\hline $\begin{array}{l}\text { ZHOU, Liqing; } \\
\text { ZHANG, Suodi; AN, } \\
\text { Young. }\end{array}$ & $\begin{array}{l}\text { Actor-network theory } \\
\text { application in global power } \\
\text { city: An empirical study }\end{array}$ & $\begin{array}{l}\text { Estuda os elementos do ecossistema de inovação, } \\
\text { incluindo universidade, empresa, conveniência de } \\
\text { tráfego, interação cultural etc., e como afetam o } \\
\text { resultado econômico em } 35 \text { cidades globais de } \\
\text { energia baseadas na teoria do ator-rede. }\end{array}$ \\
\hline BROWN & $\begin{array}{c}\text { Mission impossible? } \\
\text { Entrepreneurial universities } \\
\text { and peripheral regional } \\
\text { innovation systems }\end{array}$ & $\begin{array}{l}\text { Relação de investimentos a universidades da Escócia e } \\
\text { o retorno deste investimento a economia. Verificando } \\
\text { o alinhamento com pequenas e médias empresas, } \\
\text { empreendedores locais e com atores da política. }\end{array}$ \\
\hline $\begin{array}{l}\text { EGLASH, Stephen; } \\
\text { RIZK, Sarah. }\end{array}$ & $\begin{array}{l}\text { Discovery and application of } \\
\text { exemplary models of } \\
\text { innovation }\end{array}$ & $\begin{array}{c}\text { Explora inovações bem-sucedidas procurando } \\
\text { compreender como as universidades podem ser focos } \\
\text { de inovação e empreendedorismo e, apoia um } \\
\text { ecossistema vibrante de inovação nos campos de } \\
\text { materiais, energia e meio ambiente da Universidade } \\
\text { de Stanford. }\end{array}$ \\
\hline $\begin{array}{l}\text { GUERRERO, Maribel } \\
\text { et al. }\end{array}$ & $\begin{array}{l}\text { Entrepreneurial universities: } \\
\text { emerging models in the new } \\
\text { social and economic } \\
\text { landscape }\end{array}$ & $\begin{array}{l}\text { Examina o papel das universidades empreendedoras } \\
\text { como impulsionadoras de atividades de inovação e } \\
\text { empreendedorismo, começando com uma visão geral, } \\
\text { delineando uma estrutura abrangente. }\end{array}$ \\
\hline HAYTER, Chist & $\begin{array}{l}\text { A trajectory of early-stage } \\
\text { spinoff success: the role of } \\
\text { knowledge intermediaries } \\
\text { within an entrepreneurial } \\
\text { university ecosystem }\end{array}$ & $\begin{array}{c}\text { Compara a composição e as contribuições das redes } \\
\text { sociais entre os empresários acadêmicos e examinar } \\
\text { como essas redes coevoluem com a trajetória de } \\
\text { desenvolvimento de spin offs universitários. Além } \\
\text { disso, examina o impacto específico de políticas e } \\
\text { programas destinados a incentivar a formação de spin } \\
\text { offs universitários. }\end{array}$ \\
\hline $\begin{array}{l}\text { MCADAM, Maura; } \\
\text { MILLER, Kristel; } \\
\text { MCADAM, Rodney. }\end{array}$ & $\begin{array}{l}\text { Situated regional university } \\
\text { incubation: A multi-level } \\
\text { stakeholder perspective }\end{array}$ & $\begin{array}{l}\text { Explora os modelos de incubação universitária dentro } \\
\text { de características e restrições regionais e } \\
\text { organizacionais exclusivas e a importância no } \\
\text { desenvolvimento econômico por meio da inovação e } \\
\text { da criação de empregos. }\end{array}$ \\
\hline MILLER, Kristel et al. & $\begin{array}{l}\text { Knowledge transfer in } \\
\text { university quadruple helix }\end{array}$ & $\begin{array}{l}\text { Estuda os fenômenos da transferência de } \\
\text { conhecimento em um contexto de inovação aberta, }\end{array}$ \\
\hline
\end{tabular}




\begin{tabular}{|c|c|c|}
\hline & $\begin{array}{l}\text { ecosystems: An absorptive } \\
\text { capacity perspective }\end{array}$ & $\begin{array}{l}\text { onde múltiplos stakeholders da quádrupla hélice } \\
\text { estão interagindo. }\end{array}$ \\
\hline RANGA, Marina et al. & $\begin{array}{l}\text { Building Technology } \\
\text { Transfer Capacity in Turkish } \\
\text { Universities: } A \text { critical } \\
\text { analysis }\end{array}$ & $\begin{array}{l}\text { Analisa o retorno sobre o financiamento/investimento } \\
\text { governamental em universidades da Turquia. } \\
\text { Avaliação sobre retorno no desempenho de ensino e } \\
\text { pesquisa, criação de novos empregos, novos produtos } \\
\text { e serviços, desenvolvimento regional. }\end{array}$ \\
\hline $\begin{array}{l}\text { ROSLI, Ainurul; ROSSI, } \\
\text { Federica. }\end{array}$ & $\begin{array}{l}\text { Third-mission policy goals } \\
\text { and incentives from } \\
\text { performance-based funding: } \\
\text { Are they aligned? }\end{array}$ & $\begin{array}{l}\text { Investigação de como a política aborda o incentivo e } \\
\text { engajamento da terceira missão, o uso de } \\
\text { financiamento baseado em desempenho para } \\
\text { recompensar o sucesso das universidades, } \\
\text { alinhamento aos objetivos mais amplos de apoiar um } \\
\text { ecossistema de inovação. }\end{array}$ \\
\hline $\begin{array}{c}\text { UGNICH, Ekaterina et } \\
\text { al. }\end{array}$ & $\begin{array}{c}\text { University innovation } \\
\text { ecosystem as a mechanism } \\
\text { of innovation process } \\
\text { development }\end{array}$ & $\begin{array}{l}\text { Analisa os problemas de funcionamento do } \\
\text { ecossistema inovador da universidade. Justificando a } \\
\text { importância do ecossistema de inovação e a } \\
\text { identificação de suas perspectivas no } \\
\text { desenvolvimento da comercialização de resultados de } \\
\text { atividades intelectuais em uma instituição de ensino } \\
\text { superior. }\end{array}$ \\
\hline $\begin{array}{l}\text { MARKKULA, Markku; } \\
\text { KUNE, Hank. }\end{array}$ & $\begin{array}{l}\text { Making Smart Regions } \\
\text { Smarter: Smart } \\
\text { Specialization and the Role } \\
\text { of Universities in Regional } \\
\text { Innovation Ecosystems }\end{array}$ & $\begin{array}{c}\text { Descreve o programa da Comissão Europeia para } \\
\text { estratégias de pesquisa e inovação para especialização } \\
\text { inteligente (RIS3) e mostra como a Região de } \\
\text { Helsinque na Finlândia está usando especialização } \\
\text { inteligente, orquestração de ecossistemas e o papel } \\
\text { ativo das universidades para melhorar a inovação } \\
\text { regional. }\end{array}$ \\
\hline $\begin{array}{l}\text { STRIUKOVA, Ludmila; } \\
\text { RAYNA, Thierry. }\end{array}$ & $\begin{array}{c}\text { University-industry } \\
\text { knowledge exchange: An } \\
\text { exploratory study of Open } \\
\text { Innovation in UK universities }\end{array}$ & $\begin{array}{l}\text { Aborda a temática de open innovation dentro do } \\
\text { contexto universitário com foco no papel que as } \\
\text { universidades acreditam que devem desempenhar no } \\
\text { open innovation, bem como as mudanças que podem } \\
\text { ter surgido como consequência da maior consciência } \\
\text { das universidades sobre esse conceito. }\end{array}$ \\
\hline $\begin{array}{l}\text { CHANDRAN, } \\
\text { Govindaraju; } \\
\text { SUNDRAM, Veera; } \\
\text { SANTHIDRAN, } \\
\text { Sinnappan. }\end{array}$ & $\begin{array}{l}\text { Innovation systems in } \\
\text { Malaysia: } \text { A perspective of } \\
\text { university-industry } R \& D \\
\text { collaboration }\end{array}$ & $\begin{array}{c}\text { Trata sobre a relação entre universidade-indústria para } \\
\text { o desenvolvimento sustentável do ecossistema de } \\
\text { inovação. Mostra o compromisso do governo em } \\
\text { impulsionar a economia do país por meio da inovação. } \\
\text { Especialmente em relação à universidade em } \\
\text { colaboração industrial de P\&D. }\end{array}$ \\
\hline $\begin{array}{c}\text { MARTINEZ, Saez; } \\
\text { MORENO, Gonzalez; } \\
\text { HOGAN, Teresa. }\end{array}$ & $\begin{array}{l}\text { The role of university in eco- } \\
\text { entrepreneurship: Evidence } \\
\text { from the eurobarometer } \\
\text { survey on attitudes of } \\
\text { european entrepreneurs } \\
\text { towards eco-innovation }\end{array}$ & $\begin{array}{l}\text { Examina o impacto da colaboração universitária em } \\
\text { pequenas empresas ecoinovadoras com base nos } \\
\text { dados do primeiro inquérito sobre a ecoinovação na } \\
\text { Europa. }\end{array}$ \\
\hline BRODHAG, Christian. & $\begin{array}{c}\text { Research universities, } \\
\text { technology transfer, and job } \\
\text { creation: what infrastructure, } \\
\text { for what training? }\end{array}$ & $\begin{array}{c}\text { Analisa os ecossistemas de inovação tratando de } \\
\text { diversos aspectos desde o compromisso do negócio, } \\
\text { como o compromisso dos estudantes e das } \\
\text { universidades. }\end{array}$ \\
\hline
\end{tabular}




\begin{tabular}{|c|c|c|}
\hline ETZKOWITZ, Henry. & $\begin{array}{l}\text { StartX and the 'Paradox of } \\
\text { Success': Filling the gap in } \\
\text { Stanford's entrepreneurial } \\
\text { culture }\end{array}$ & $\begin{array}{c}\text { Identifica a capacidade empreendedora e de } \\
\text { transferência de tecnologia. }\end{array}$ \\
\hline ZHANG, Linxiu et al. & $\begin{array}{l}\text { Are elite university } \\
\text { graduates aiding China's } \\
\text { transition to an innovation- } \\
\text { based economy? Results } \\
\text { from a career choices survey } \\
\text { among would-be innovators } \\
\text { in China and the USA }\end{array}$ & $\begin{array}{l}\text { Estudo de caso com alunos matriculados nos } \\
\text { departamentos de engenharia das universidades de } \\
\text { elite na China e nos EUA. Espera-se que a análise possa } \\
\text { emprestar alguma base empírica para descobertas no } \\
\text { debate em curso sobre a China e o seu potencial de } \\
\text { inovação. }\end{array}$ \\
\hline $\begin{array}{l}\text { HARRISON, Kenneth } \\
\text { et al. }\end{array}$ & $\begin{array}{l}\text { Building a life sciences } \\
\text { innovation ecosystem }\end{array}$ & $\begin{array}{l}\text { Discute a importância de gerar inovação na área de life } \\
\text { science e criação de startup. }\end{array}$ \\
\hline $\begin{array}{l}\text { CARAYANNIS, Elias; } \\
\text { CAMPBELL, David. }\end{array}$ & $\begin{array}{c}\text { Open Innovation Diplomacy } \\
\text { and a 21st Century Fractal } \\
\text { Research, Education and } \\
\text { Innovation (FREIE) } \\
\text { Ecosystem: Building on the } \\
\text { Quadruple and Quintuple } \\
\text { Helix Innovation Concepts } \\
\text { and the "Mode 3" } \\
\text { Knowledge Production } \\
\text { System }\end{array}$ & $\begin{array}{l}\text { Trata sobre diversos temas como a Diplomacia, } \\
\text { Democracia Cultural, Níveis de modos e sobre tríplice } \\
\text { hélice, quádrupla hélice e quíntupla hélice. Sugerindo } \\
\text { uma ligação entre teoria dos sistemas e a } \\
\text { compreensão do conhecimento, enfatizando sistemas } \\
\text { multiníveis de conhecimento, com abordagem para } \\
\text { criação, difusão e uso do conhecimento. }\end{array}$ \\
\hline $\begin{array}{l}\text { MAES, Katrien; } \\
\text { DEBACKERE, } \\
\text { Koenraad; VAN DUN, } \\
\text { Paul. }\end{array}$ & $\begin{array}{l}\text { Universities, research and } \\
\text { the "innovation Union" }\end{array}$ & $\begin{array}{l}\text { Apresenta os pontos de vista de algumas das } \\
\text { principais universidades de investigação intensiva } \\
\text { (RIUs) da Europa, como contributo para o } \\
\text { desenvolvimento da estratégia da união europeia (UE) } \\
\text { e as iniciativas que dela resultam. }\end{array}$ \\
\hline $\begin{array}{l}\text { MERCAN, Birol; } \\
\text { GOKTAS, Din. }\end{array}$ & $\begin{array}{c}\text { Components of innovation } \\
\text { ecosystems: A cross-country } \\
\text { study }\end{array}$ & $\begin{array}{l}\text { Analisa os componentes determinantes para um } \\
\text { Ecossistema de Inovação segundo: desenvolvimento } \\
\text { de clustering, a colaboração universidade-indústria e a } \\
\text { cultura para inovar. }\end{array}$ \\
\hline $\begin{array}{l}\text { TRAILER, Helmut; } \\
\text { WATZKE, Heribert; } \\
\text { SAGUY, Sam. }\end{array}$ & $\begin{array}{l}\text { Reinventing R\&D in an Open } \\
\text { Innovation Ecosystem }\end{array}$ & $\begin{array}{l}\text { Estudo de caso sobre a mudança de paradigma para a } \\
\text { reinvenção da P\&D. }\end{array}$ \\
\hline $\begin{array}{l}\text { COSH, Andy; } \\
\text { HUGHES, Alan. }\end{array}$ & $\begin{array}{c}\text { Never mind the quality feel } \\
\text { the width: University- } \\
\text { industry links and } \\
\text { government financial } \\
\text { support for innovation in } \\
\text { small high-technology } \\
\text { businesses in the UK and the } \\
\text { USA }\end{array}$ & $\begin{array}{l}\text { Análise de uma amostra de empresas britânicas e } \\
\text { americanas nos setores de serviço e manufatura, a fim } \\
\text { de analisar a força relativa dos ecossistemas (relação } \\
\text { entre indústria e universidade). }\end{array}$ \\
\hline SMITH, Kenneth. & $\begin{array}{c}\text { Building an Innovation } \\
\text { Ecosystem: Process, Culture } \\
\text { and Competencies }\end{array}$ & $\begin{array}{c}\text { Examina os três componentes de um ecossistema de } \\
\text { inovação (empresas, universidades e governo) e suas } \\
\text { implicações para corporações, universidades e } \\
\text { políticas públicas. }\end{array}$ \\
\hline
\end{tabular}

Fonte: Elaborado pelos autores (2019). 
Na classificação da base teórica dos 34 estudos em assuntos centrais associados a que está sendo discutido sobre Universidades e o Ecossistema de Inovação, empregou-se análise de conteúdo com a leitura integral das obras selecionando o foco dominante de cada referência, como pode ser visto no Quadro 5.

Quadro 5 - Assuntos centrais dos artigos analisados

\begin{tabular}{|c|c|}
\hline Autores & $\begin{array}{l}\text { Assuntos centrais do portfólio } \\
\text { bibliográfico }\end{array}$ \\
\hline $\begin{array}{l}\text { MARKKULA, Markku; KUNE, Hank (2015); UGNICH, Ekaterina } \\
\text { et al. (2016); KIVIMAA, Paula; BOON, Wouter; ANTIKAINEN, } \\
\text { Riina (2017); MAES, Katrien; DEBACKERE, Koenraad; VAN } \\
\text { DUN, Paul (2011); ZHOU, Liqing; ZHANG, Suodi; AN, Young } \\
\text { (2017); BRODHAG, Christian. (2013); CHEN, Shih; LIN, Wei } \\
\text { (2017); MILLER, Kristel et al. (2016) }\end{array}$ & $\begin{array}{l}\text { Universidades no Ecossistema de } \\
\qquad \text { Inovação }\end{array}$ \\
\hline $\begin{array}{l}\text { GUERRERO, Maribel et al. (2016); ETZKOWITZ, Henry (2013); } \\
\text { EGLASH, Stephen; RIZK, Sarah (2016); HAYTER, Chistopher } \\
\text { (2016); HARRISON, Kenneth et al. (2012); THEODORAKI, } \\
\text { Christina; MESSEGHEM, Karim; RICE, P. (2018); MCADAM, } \\
\text { Maura; MILLER, Kristel; MCADAM, Rodney (2016); ZHANG, } \\
\text { Linxiu et al. (2013) }\end{array}$ & $\begin{array}{l}\text { Universidades empreendedoras } \\
\text { (criação de spin-off, startup, } \\
\text { incubadoras universitárias, perfil } \\
\text { universitário inovador, atividades de } \\
\text { empreendedorismo) }\end{array}$ \\
\hline $\begin{array}{l}\text { THEODORAKI, Christina; MESSEGHEM, Karim; RICE, P. } \\
\text { (2018); HAYTER, Chistopher. (2016); }\end{array}$ & $\begin{array}{l}\text { Ecossistema universitário } \\
\text { empreendedor }\end{array}$ \\
\hline $\begin{array}{c}\text { AUDY, Jorge (2017); BROWN, Roger (2016); ROSLI, Ainurul; } \\
\text { ROSSI, Federica (2016) }\end{array}$ & Terceira missão da universidade. \\
\hline $\begin{array}{c}\text { AUDY, Jorge (2017); DANIELS, Chux; USTYUZHANTSEVA, } \\
\text { Olga; YAO, Wei (2017); CARAYANNIS, Elias; CAMPBELL, } \\
\text { David (2011); SMITH, Kenneth (2006) }\end{array}$ & Tríplice Hélice \\
\hline $\begin{array}{l}\text { DEL VECCHIO, Pasquale et al. (2017); MILLER, Kristel et al. } \\
\text { (2016); CARAYANNIS, Elias; CAMPBELL, David (2011) }\end{array}$ & Quádrupla Hélice \\
\hline CARAYANNIS, Elias; CAMPBELL, David (2011) & Quíntupla Hélice \\
\hline $\begin{array}{c}\text { RANGA, Marina et al. (2017); CHANDRAN, Govindaraju; } \\
\text { SUNDRAM, Veera; SANTHIDRAN, Sinnappan (2014); } \\
\text { MARTINEZ, Saez; MORENO, Gonzalez; HOGAN, Teresa } \\
\text { (2014); MERCAN, Birol; GOKTAS, Din (2011); TRAILER, } \\
\text { Helmut; WATZKE, Heribert; SAGUY, Sam (2011); COSH, } \\
\text { Andy; HUGHES, Alan (2010); GALAN-MUROS, Victoria et al. } \\
(2017)\end{array}$ & Relação universidade-indústria \\
\hline BRANCO, Jose; LIMA, Celso (2018); & $\begin{array}{c}\text { Ecossistemas de inovação baseado em } \\
\text { fluxos de conhecimento ao contexto } \\
\text { universitário }\end{array}$ \\
\hline DEL VECCHIO, Pasquale et al. (2017) & $\begin{array}{l}\text { Living } L a b \text {, criação de Ecossistemas e } \\
\text { redes dinâmicas de inovação }\end{array}$ \\
\hline HU, Lina; LI, Yali; ZHANG, Bin (2017) & $\begin{array}{l}\text { Tipos de inovação relacionados à } \\
\text { universidade e gestão pública }\end{array}$ \\
\hline BROWN, Roger (2016); RANGA, Marina et al. (2016) & $\begin{array}{c}\text { Retorno de investimento } \\
\text { governamental com foco em inovação } \\
\text { em universidades }\end{array}$ \\
\hline STRIUKOVA, Ludmila; RAYNA, Thierry (2015) & $\begin{array}{c}\text { Open innovation dentro do contexto } \\
\text { universitário }\end{array}$ \\
\hline
\end{tabular}

Fonte: Elaborado pelos autores (2019). 
Com a análise dos assuntos centrais verificou-se a concentração maior de autores tratando do tema Universidades no Ecossistema de Inovação discutindo o papel dinâmico das universidades ao interagir com os outros atores do ecossistema de inovação. Chen e Lin (2017), destacam que a universidade além de disseminar o conhecimento ela é intermediária da comercialização de tecnologia. Neste contexto as universidades assumem seu papel de empreendedoras, passando a controlar e comercializar o conhecimento gerado/propriedade intelectual, principalmente em países desenvolvidos, desempenhando assim além de (1) pesquisa e (2) ensino o papel da "terceira missão" (ETZKOWITZ; LEYDESDORFF, 2000).

Como caraterística da terceira missão da universidade, pode-se citar a criação de spin offs universitárias, startup, incubadoras universitárias, desenvolvimento de perfil universitário inovador, atividades de empreendedorismo em geral. Hayter (2016), examina o impacto específico de políticas e programas destinados a incentivar a formação e o sucesso de spin offs universitárias. $O$ autor salienta que o sucesso do spin offdepende de contatos acadêmicos e não acadêmicos que conectem professores e alunos a outras redes sociais, logo, as contribuições das universidades dependem da existência e da inter-relação de intermediários de conhecimento heterogêneos, pouco coordenados, guiados por um senso de coletividade para encorajar e apoiar o empreendedorismo acadêmico.

Apesar do papel importante que as universidades desempenham na cadeia de inovação, como provedoras e disseminadoras de conhecimento, elas não atuam sozinhas, ao contrário fazem parte de um ecossistema de inovação maior que envolve atores como governo e indústria (MAES; DEBACKERE; VAN DUN, 2011).

Para Smith (2006), a triple hélice faz relação com três "hélices" que se entrelaçam gerando um sistema nacional de inovação envolvendo academia/universidades, indústria e governo/estado. Neste contexto, a quádrupla hélice, significa a junção de uma "quarta hélice" que identifica em cultura pública, onde existe a participação efetiva da sociedade civil (MILLER et al., 2016). Carayannis e Campbell (2011), destacam que a quíntupla hélice traz a perspectiva dos ambientes naturais da sociedade e da economia para a produção de conhecimento e de sistemas de inovação. No entanto, o autor enfatiza que os ambientes devem ser conceituados como impulsionadores para o avanço de produção do conhecimento e sistemas de inovação compatível com os interesses analíticos relacionados a ecologia.

De acordo com Chandran, Sundram e Santhidran, (2014) as universidades veem as indústrias como uma importante fonte de conhecimento. A força da colaboração universidade-indústria é a dimensão mais importante de um ecossistema de inovação e, essa interação acelera a inovação e proporciona benefícios econômicos e intelectuais para ambos os envolvidos (MERCAN; GOKTAS, 2011).

\section{CONCLUSÃO}

A revisão da literatura permite o desenvolvimento e a compreensão do estado da arte do tema pesquisado, além de contribuir com a geração de conhecimento já existente sobre a produção científica (VAZ; LEZANA; MALDONADO, 2017).

Foi realizada uma revisão estruturada de literatura no período de 2006 e 2018 com busca nas bases de dados Web of Science e Scopus, conforme método apresentado de Construção da coleção de artigos, Processo de filtragens, Análise bibliométrica e Análise conteúdo. Possibilitando a análise quantitativa e qualitativa do portfólio bibliográfico composto por 34 artigos estabelecendo um panorama das pesquisas realizadas nos últimos no tema Universidade e ecossistema de inovação.

A análise bibliométrica resultou no parecer quantitativo dos 34 artigos do portfólio bibliográfico, sendo identificado que foram escritos por 92 autores e coautores, publicados em 30 periódicos no período de 2006 a 2018, em 22 países, por 62 instituições de ensino e, apresentam um total de 69 palavras-chave com destaque para universitye innovation.

A partir da análise de conteúdo, verificou-se que os clusters em geral, possuem autores e coautores que estão abordando o mesmo tema central "universidade e ecossistema de inovação". Os assuntos abordados possuem diferentes ligações entre universidade e autores do ecossistema, principalmente considerando a relação do governo e indústria. Alguns autores como Harrison et al. (2012), Etzkowitz (2013), Zhang et al. 
(2013), Eglash e Rizk (2016), Guerrero et al. (2016), Hayter (2016), McAdam, Miller e McAdam (2016) e Theodoraki, Messeghem e Rice (2018), e destacam a comercialização do conhecimento científico para além dos limites da universidade, destacando barreiras e oportunidades e o papel da universidade empreendedora, como por exemplo, na criação de spin offs, startups, incubadoras e aceleradoras, entre outras ações voltadas ao cenário empreendedor.

Portanto, as universidades precisam criar uma atmosfera adequada em que a comunidade universitária possa explorar, avaliar e potencializar o conhecimento, para que o mesmo seja transformado e aplicado de acordo com a realidade da sociedade.

Com isso, espera-se que este trabalho influencie na realização de novas pesquisas para que visem compreender as diferentes realidades das universidades, com o intuito de identificar possíveis caminhos de crescimento e fomentar a cooperação no desenvolvimento de projetos inovadores, criando e disseminando ainda mais conhecimento, pois além de estimular, as universidades devem dar apoio e capacitação às iniciativas empreendedoras, para que uma haja uma real integração ao ecossistema de inovação.

Como recomendação para os trabalhos futuros, sugere-se: i) utilizar um número maior de bancos de dados do portal da Capes; ii) considerar outros trabalhos, como teses, dissertações, monografias, congressos e livros e iv) identificar outros artigos alinhados ao tema nas referências dos artigos retornados. Logo, com o desenvolvimento deste trabalho, pôde-se contribuir com um panorama da produção acadêmica e fundamentar novos estudos que abordam o relacionamento das universidades com os demais atores do ecossistema de inovação.

\section{REFERÊNCIAS}

AKOIJAM, Amitkumar Singh; KRISHNA, Venni. Exploring the Jawaharlal Nehru National Solar Mission (JNNSM): Impact on innovation ecosystem in India. African Journal of Science, Technology, Innovation and Development, v. 9, p. 573-585. 2017.

ARNKIL, Robert et al. Exploring quadruple helix - outlining user oriented innovation models. Tampere: University of Tampere, Institute for Social Research, Work Research Centre. 2010.

ASHEIM, Bjorn; GERTLER, Meric. The geography of innovation: Regional innovation systems. In: FAGERBERG, Jan; MOWERY, David; NELSON. The Oxford Handbook of Innovation. Oxford University Press, Oxford, 2005. p. 291-317.

AUDRESTSCH, David. Firm growth and innovation. Small Business Economics, v. 43, n. 4, p. 743-749, 2014.

AUDY, Jorge. A inovação, o desenvolvimento e o papel da Universidade. Estudos Avançados, n. 31, p. 75-87, 2017.

BRANCO, Jose; LIMA, Celso. Univeristies as Knowledge Flow Based Innovation Ecosystems. Revista Praxis Educacional, v. 14, n. 27, p. 84-108, 2018.

BRENNER, Thomas et al. Regional innovation systems, clusters, and knowledge networking, Papers in regional science, v. 90 n. 2, p. 243-249, 2011.

BRODHAG, Christian. Research universities, technology transfer, and job creation: what infrastructure, for what training? Studies in Higher Education, v. 38, p. 388-404, 2013.

BROWN, Roger. Mission impossible? Entrepreneurial universities and peripheral regional innovation systems. Industry and Innovation, v. 23, p. 189-205, 2016. 
CARAYANNIS, Elias; CAMPBELL, David. Open Innovation Diplomacy and a 21st Century Fractal Research, Education and Innovation (FREIE) Ecosystem: Building on the Quadruple and Quintuple Helix Innovation Concepts and the "Mode 3" Knowledge. Journal Production System, n. 2, p. 327-372, 2011.

CHANDRAN, Govindaraju; SUNDRAM, Veera; SANTHIDRAN, Sinnappan. Innovation systems in Malaysia: A perspective of university-industry R\&D collaboration. Al and Society, v. 29, p. 435-444. 2014.

CHEN, Shih; LIN, Wei. The dynamic role of universities in developing an emerging sector: a case study of the biotechnology sector. Technological Forecasting and Social Change, v. 123, p. 283-297. 2017.

COOKE, Philip; MORGAN, Kevin. The Associational Economy: Firms, Regions and Innovation. Oxford: Oxford University Press, 1998.

$\mathrm{COSH}$, Andy; HUGHES, Alan. Never mind the quality feel the width: University-industry links and government financial support for innovation in small high-technology businesses in the UK and the USA. Journal of Technology Transfer, n. 35, p. 66-91. 2010.

CYERT, Richard; GOODMAN, Paul. Creating effective university-industry alliances: an organizational learning perspective, Organizational Dynamics, v. 26, n. 4, p. 45-57, 1997.

DANIELS, Chux; USTYUZHANTSEVA, Olga; YAO, Wei. Innovation for inclusive development, public policy support and triple helix: perspectives from BRICS. African Journal of Science Technology Innovation \& Development, n. 9, p. 513-527. 2017.

DEL VECCHIO, Pasquale et al. Living Lab as an Approach to Activate Dynamic Innovation Ecosystems and Networks: An Empirical Study. International Journal of Innovation and Technology Management, v. 14, n. 5. 2017.

D'ESTE, Peter; PATEL, Parimal. University-industry linkages in the UK: What are the factors underlying the variety of interactions with industry? Research Policy. v. 36, n. 9, p. 1295-1313, 2007.

EDQUIST, Charles. Systems of innovation: Perspectives and challenges. In: FAGERBERG, Jan; MOWERY, David; NELSON (org.). The Oxford Handbook of Innovation. Oxford: Oxford Handbooks, 2005. p. 181-208.

EGLASH, Stephen; RIZK, Sarah. Discovery and application of exemplary models of innovation. MRS Bulletin, v. 41, n. 6, p. 479-487. 2016.

ENDNOTE. Compatibility and System Requirements. [2011]. Disponível em: https://endnote.com/productdetails/compatibility/. Acesso em: 21 ago. 2019.

ETZKOWITZ, Henry; LEYDESDORFF, Loet. The dynamics of innovation: from National Systems and "Mode 2" to a Triple Helix of university-industry-government relations. Res. Policy, v. 29, n. 2, p. 109-123, 2000.

ETZKOWITZ, Henry; KLOFSTEN, Magnus. The innovating region: Toward a theory of knowledgebased regional development. R\&D Management, v. 35, p. 243-255. 2005.

ETZKOWITZ, Henry. StartX and the 'Paradox of Success': Filling the gap in Stanford's entrepreneurial culture. Social Science Information, v. 52, n. 4, p. 605-627. 2013.

FREEMAN, Christopher. Technology, policy, and economic performance: Lessons from Japan. London and New York: Pinter Publishers, 1987.

FUMASOLI, Tatiana; PINHEIRO, Romulo; STENSAKER, Bjorn. Strategizing Identity in Higher Education, ISL Working Paper, n. 3, 2014. 
GALVÃO, Cristina; SAWADA, Namie; TREVIZAN, Maria. Revisão sistemática: recurso que proporciona a incorporação das evidências na prática da enfermagem. Rev. Latino-Am. Enfermagem, v. 12, n. 3, p. 549556, 2004.

GALAN-MUROS, Victoria et al. Nurture over nature: How do European universities support their collaboration with business? Journal of Technology Transfer, v. 42, n.1, p. 184-205, 2017.

GEROLAMO, Mateus Cecílio et al. Performance management of regional clusters and SME cooperation networks, International Journal of Business Excellence, v. 1 n. 4, p. 457-483, 2008.

GIL, Antônio. Métodos e técnicas de pesquisa social. 6. ed. São Paulo: Atlas SA, 2008.

GRADY, Razak; PRATT, Jonh. The UK Technology Transfer System: Calls for Stronger Links between Higher Education and Industry, Journal of Technology Transfer, v. 25, n. 2, p. 205-211, 2000.

GUERRERO, Maribel et al. Entrepreneurial universities: emerging models in the new social and economic landscape. Small Business Economics, v. 47, n. 3, p. 551-563. 2016.

HARMAN, Grant. University-industry research partnerships in Australia: extent, benefits and risks, Higher Education Research and Development, v. 20 n. 3, p. 245-264, 2001.

HARRISON, Kenneth et al. Building a life sciences innovation ecosystem. Science Translational Medicine, v. 4, n. 157, p. 37, 2012.

HAYTER, Chistopher. A trajectory of early-stage spinoff success: the role of knowledge intermediaries within an entrepreneurial university ecosystem. Small Business Economics, v. 47, n. 3, p. 633-656. 2016.

HOWLETT, Robert. Knowledge Transfer between UK Universities and Business' In Innovation through Knowledge Transfer: Smart Innovation, Systems and Technologies, Springer-Verlag, v. 10, n. 5, p. 1-16. 2010.

HU, Lina; LI, Yali; ZHANG, Bin. Study on university scientific innovation and cultural construction from the perspective of public management. Revista de la Facultad de Ingenieria, v. 32, n. 12, p. 970-976. 2017.

JACOBS, Jane. The Economy of Cities. New York: Vintage, 1969.

KIVIMAA, Paula; BOON, Wouter; ANTIKAINEN, Riina. Commercialising university inventions for sustainability-a case study of (non-)intermediating 'cleantech' at Aalto University. Science and Public Policy, v. 44, n. 5, p. 631-644. 2017.

KRUSS, Glenda et al. Promoting higher education-industry partnerships and collaborations. South Africa: Research and Innovation Strategy Group Higher Education, 2011.

LEE, Young. The sustainability of university-industry research collaboration: an empirical assessment, Journal of Technology Transfer, v. 25 n. 2, p. 111-133, 2010.

LIMA, Mauricio; SOARES, Bernardo; BACALTCHUK, Josué. Psiquiatria baseada em evidências. Revista Brasileira de Psiquiatria, v. 22, n. 3, p. 142- 146, 2000.

LOCKETT, Andy; WRIGHT, Mike; WILD, Andrew. The Institutionalization of Third Stream Activities in UK Higher Education: The Role of Discourse and Metrics, British Journal of Management, n. 26, p. 78-92, 2014.

LUNDVALL, Bengt. Product Innovation and User-Producer Interaction. Industrial Development Research Series, n. 31, p. 39, 1985. 
MAES, Katrien; DEBACKERE, Koenraad; VAN DUN, Paul. Universities, research and the "innovation Union". Procedia - Social and Behavioral Sciences, n. 13, p. 101-116. 2011.

MALERBA, Franco. Sectoral systems of innovation: Concepts, issues, and analyses of six major sectors in Europe. Cambridge: Cambridge University Press, 2004.

MARKKULA, Markku; KUNE, Hank. Making Smart Regions Smarter: Smart Specialization and the Role of Universities in Regional Innovation Ecosystems. Technology Innovation Management Review, v. 5, n. 10, p. 7-15, 2015.

MARTINEZ, Saez; MORENO, Gonzalez; HOGAN, Teresa. The role of university in eco-entrepreneurship: Evidence from the eurobarometer survey on attitudes of european entrepreneurs towards eco-innovation. Environmental Engineering and Management Journal, v. 13, n. 10, p. 25, 2014.

MCADAM, Maura; MILLER, Kristel; MCADAM, Rodney. Situated regional university incubation: A multi-level stakeholder perspective. Technovation, v. 50, p. 69-78, 2016.

MERCAN, Birol; GOKTAS, Din. Components of innovation ecosystems: A cross-country study. International Research Journal of Finance and Economics, n. 76, p. 102-112. 2011.

MILLER, Kristel et al. Knowledge transfer in university quadruple helix ecosystems: An absorptive capacity perspective. R and D Management, v. 46, n. 2, p. 383-399, 2016.

MOWERY, David; SAMPAT, Bhaven. Universities in national innovation systems. In: FAGERBERG, Jan; MOWERY, David; NELSON (org.). The oxford handbook of innovation. Oxford: Oxford University Press, 2005. p. 209-239.

OECD. The measurement of scientific and technological activities: proposed guidelines for collecting and interpreting technological innovation data. 3rd. ed. Paris: OECD Publishing, 2005. E-book.

PHAN, Philip; SIEGEL, Donald. The effectiveness of university technology transfer: Lessons learned from quantitative and qualitative research in the US and the UK. Foundations and Trends in Entrepreneurship, v. 2, n. 2, p. 1-53, 2005.

PELLEGRIN, Ivan et al. Redes de inovação: construção e gestão da cooperação pró-inovação. Revista de Administração-RAUSP, v. 42, n. 3, p.313-325. 2007.

POWELL, Walter; KOPUT, Koput; SMITH, Laurel. Interorganizational collaboration and the locus of control of innovation: Networks of learning in biotechnology. Administrative Science Quarterly, n. 41, p. 116-145, 1996.

RANGA. Marina; MROCZOWSKI, Tomasz; ARAISO, Tsunehisa. University-industry cooperation and the transition to innovation ecosystems in Japan. Industry and Higher Education, v. 31, n. 6, p. 373-387, 2017.

RANGA, Marina et al. Building Technology Transfer Capacity in Turkish Universities: A critical analysis. European Journal of Education, v. 51, n.1, p. 90-106. 2016.

ROMANO, Andy; PASSIANTE, George; DEL VECCHIO, Pasquale. Knowledge-intensive Entrepreneurship in Innovation Ecosystems: towards the conceptual model of a Regional Entrepreneurial Learning Center, v. 16, n. 1, p. 24-61, 2014.

ROSLI, Ainurul; ROSSI, Federica. Third-mission policy goals and incentives from performance-based funding: Are they aligned? Research Evaluation, v. 25, n. 4, p. 427-441, 2016. 
SANTORO, Michael; CHAKRABARTI, Alok. Firm size and technology centrality in industry-university interactions. Research Policy, v. 31, n. 7, p. 1163-1180, 2002.

SCHARTINGER, Doris et al. Knowledge interactions between universities and industry in Austria: sectorial patterns and determinants, Research Policy, v. 31 n. 3, p. 303-328, 2002.

SCHUMPETER, Joseph. Business Cycles: A Theoretical, Historical and Statistical Analysis of the Capitalist Proces. New York: McGraw Hill, 1939.

SMITH, Kenneth. Building an Innovation Ecosystem: Process, Culture and Competencies. Industry and Higher Education, v. 20, n. 4, p. 219-224, 2006.

STRIUKOVA, Ludmila; RAYNA, Thierry. University-industry knowledge exchange: An exploratory study of Open Innovation in UK universities. European Journal of Innovation Management, v. 18, n. 4, p. 471-492, 2015.

TOMAÉL, Maria Inês; MARTELETO, Regina Maria. Redes sociais: posições dos atores no fluxo da informação 10.5007/1518-2924.2006v11 nesp1 p75. Encontros Bibli: revista eletrônica de biblioteconomia e ciência da informação, Florianópolis, p. 75-91, jul. 2006. ISSN 1518-2924. Disponível em:

https://periodicos.ufsc.br/index.php/eb/article/view/1518-2924.2006v11 nesp1 p75. Acesso em: 21 ago. 2019. doi:https://doi.org/10.5007/1518-2924.2006v11nesp1p75.

THEODORAKI, Christina; MESSEGHEM, Karim; RICE, P. Mark. A social capital approach to the development of sustainable entrepreneurial ecosystems: an explorative study. Small Business Economics. v. 51, n. 1, p. 153$170,2018$.

TRAILER, Helmut; WATZKE, Heribert; SAGUY, Sam. Reinventing R\&D in an Open Innovation Ecosystem. Journal of Food Science, v. 76, n. 2, p. 62-68, 2011.

UGNICH, Ekaterina et al. University innovation ecosystem as a mechanism of innovation process development. Social Sciences (Pakistan), v. 11, n. 14, p. 3479-3483, 2016.

URBANO, David; GUERRERO, Maribel. Entrepreneurial universities: Socio-economic impacts of academic entrepreneurship in a European region. Economic Development Quarterly, v. 27, n. 1, p. 40-55, 2013.

VAZ, C. R.; LEZANA, A. G. R.; MALDONADO, U. M. Mapeamento Bibliometrico da Literatura Cientifica de Ecoinovação (1978-2017). SIMPOI-Fgv. 2017.

VEUGELERSA, Reinhilde; CASSIMAN, Bruno. R\&D cooperation between firms and universities. Some empirical evidence from Belgian manufacturing, International Journal of Industrial Organization, v. 23, n 5/6, p. 355-379, 2005.

VORLEY, Tim; LAWTON, Hellen. Universities and the Knowledgebased Economy, Environment and Planning C: Government and Policy, v. 25, n. 6, p. 775-778, 2007.

ZAJAC, Edward; OLSEN, Cyrus. From transaction cost to transactional value analysis: Implications for the study of interorganizational strategies. Journal of Management Studies, n. 30, p. 131-145, 1993.

ZHANG, Linxiu et al. Are elite university graduates aiding China's transition to an innovation-based economy? Results from a career choices survey among would-be innovators in China and the USA. Asia-Pacific Journal of Accou. v. 20, p. 58-69, 2013.

ZHOU, Liqing; ZHANG, Suodi; AN, Young. Actor-network theory application in global power city: An empirical study. Revista de la Facultad de Ingenieria, v. 32, n. 12, p. 200-209, 2017. 